Master's Thesis

Computer Science

January 2013

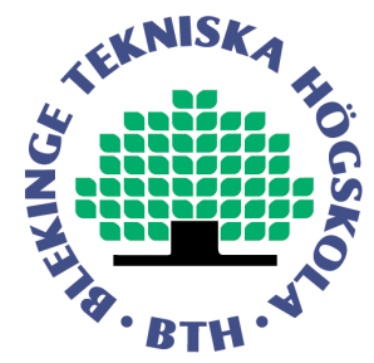

\title{
Virtual Firewalling For Migrating Virtual Machines In Cloud Computing
}

\author{
Mahwish Anwar
}


This thesis is submitted to the School of Computing at Blekinge Institute of Technology on the partial fulfillment of the requirements for the degree of Master of Science in Computer Science. The thesis is equivalent to 20 weeks of full time studies (30 ECTS).

\section{Contact Information:}

Author:

Mahwish Anwar

E-mail: mahwish.anwar@gmail.com

University advisor:

Dr. David Erman, Ph.D., Telecommunication Systems

School of Computing

Blekinge Institute of Technology

In co-operation with:

Klas Nyström, Managing Director

HiQ Karlsrona AB

Address: Campus Grasvik 4, SE-371 75 Karlskrona, Sweden.

Phone: +46455617627

Magnus Bergman, Chief Technical Officer

City Network Hosting AB

Address: Borgmastaregatan 18, SE-371 34 Karlskrona, Sweden.

Phone: +46455690000

School of Computing

Blekinge Institute of Technology

SE - 37179 Karlskrona

Sweden
Internet : www.bth.se/com

Phone : +46455385000

Fax : : +46455385057 


\begin{abstract}
Context. Cloud Computing (CC) uses virtualization to provide computing resources on demand via Internet. Small and large organizations benefit from CC because of reduced operating costs and increase in business agility. The migrating Virtual Machine (VM) is vulnerable from attacks such as fake migration initiations, service interruptions, manipulation of data or other network attacks. During live migration any security lax in VM firewall policy can put the VM data, OS and the applications on it at risk. A malicious VM can pose threat to other VMs in its host and consequently for VMs in LAN. Hardware firewalls only protect VM before and after migration. Plus, they are blind to virtual traffic. Hence, virtual firewalls (VFs) are used to secure VMs. Mostly; they are deployed at Virtual Machine Monitor-level (VMM) under Cloud provider's control. Source VMM-level VF provides security to VM before the migration incurs and the destination VMM-level VF starts securing VM after migration is completed. It thus, becomes possible for attacker to use the intermediate migrating window to launch attacks on VM. Considering the potential of VFs there should be a great value in using open source VFs at VM-level for protecting VMs during migration, thereby, reducing the attacker's slot to gain access to VM. It would enable hardened security for overall VM migration.
\end{abstract}

Objectives. The aim is to investigate VM-level firewalling using open source firewall as a complementary security layer to VMM-level firewalling, to secure migrating VM in the CC domain. The first objective is to identify how virtual firewalls secure migrating VM in CC and to propose VMlevel open-source virtual firewalling for protecting VM during migration. Later the VF is implemented to validate and evaluate its intactness or activeness during migration in real Cloud data center.

Methods. In the literary review 9 electronic libraries are used, which include IEEE Xplore, ACM Digital Library, SCOPUS, Engineering Village and Web of Knowledge. Studies are selected after querying libraries for 2 key terms 'virtual machine' and 'migration' (along with other variations/synonyms), in the abstract. Relevant papers on the subject are read and analyzed. Finally, the information gaps are identified. Using a lacuna the experimental solution is designed. To test the potential of VF at VM-level for migrating VM's security the experimental validation is performed using stratification samples of firewall rules. The VF evaluation is done using continuous ICMP echo packet transmission. The packets are analyzed to determine firewall behavior during migration. To evaluate the validity, the VM migration is performed 8 times in City Network data center.

Results. The literary review identified the widespread use of VMM-level firewalling for migrating VM's security in CC. The VM-level VFs were not researched nor evaluated for intactness during migration. The experiment performed at City Network demonstrated that the VM-level VF secures VM during migration (on average) for $96 \%$ of migration time, thereby reducing attack window for attacker during VM mobility. According to the results the average total migration time was $16.6 \mathrm{~s}$ and average downtime of firewall was as low as $0.47 \mathrm{~s}$, which means that VF at VM-level protects VM during entire migration span except when VM's down (4\% of migration time).

Conclusions. The research concludes that VM-level firewalling using open source VF as an additional security layer in $\mathrm{CC}$ for $\mathrm{VM}$ migrations is feasible to employ and will enhance the migrating machine's security by providing hardened firewall service during migration process, thus, reducing the potential attack window. VMM-level VF provides security in post and pre migration phase. Using VM-level VF as a complementary measure to VMM-level VF enables additional protection for VM migration process, thereby reducing the chances for attacker to attack VM during transition.

Keywords: virtual machine, migration, firewall. 


\section{ACKNOWLEDGEMENT}

Firstly, I would like to express gratitude to my supervisor, Dr. David Erman, for his valuable recommendations and assistance during the research work. His generosity in giving time for discussions is greatly appreciated.

I would like to acknowledge the invaluable support of HiQ for the duration of the research. I am obliged to Klas Nyström, the Managing Director at HiQ Karlskrona AB, for being supportive throughout the research and helping me to connect to the companies in Sweden.

This research would not have been possible without the cooperation of City Network. I like to acknowledge in particular the time put in by Magnus Bergman, the Chief Technical Officer at City Network, for giving me insight on the Cloud industry and also for lending the technological assistance in executing the research idea.

I am also indebted to Stefan Bernbo, Chief Executive Officer at Compuverde AB, for being willing to share his knowledge and for enlightening me about current data center trends.

I am grateful to my colleagues and friends for their useful ideas and constant encouragement. Many thanks to Sogand Shirinbab, Ezedin Essafa, Poornima Ravi and Binta Jammeh.

The road to my post-graduation has been long. Two people, whose presence made the completion of research possible, are my parents. My love and prayers to Ami and Baba, for having confidence in me, and supporting me in every way. They have helped me more than they can know. My special thanks and love to Haad Tariq, my husband and best-friend for his unfailing encouragement, love and support throughout my research and past years. My siblings, Dania Anwar, Farheen Anwar, Naveed Anwar and Ayesha Anwar, I thank you all for adding cheers and hope.

This acknowledgement would be incomplete without crediting all my teachers, colleagues and friends. Special thanks to Dr. Martin Boldt, Dr. Bengt Carlsson and Dr. Samuel A. Fricker for their useful feedback on initial idea generation. 


\section{CONTENTS}

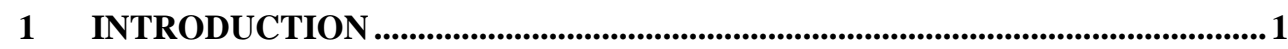

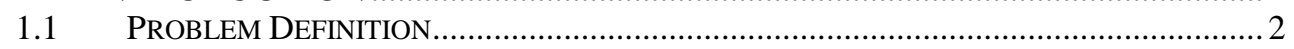

1.2 RESEARCH QUESTIONS ……………………………................................... 3

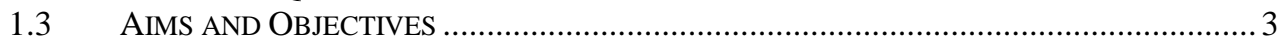

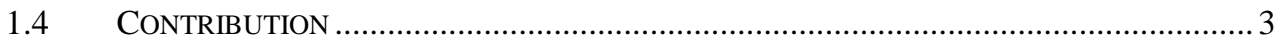

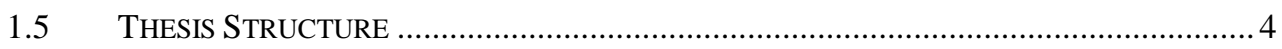

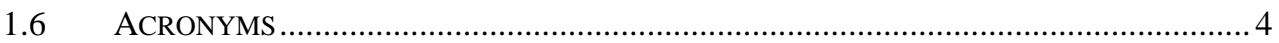

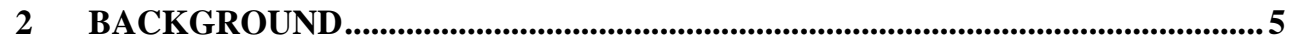

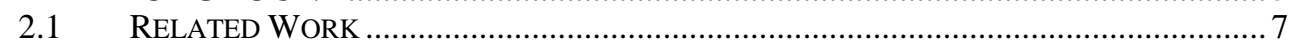

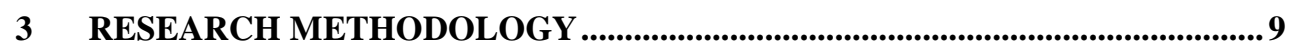

3.1 SYSTEMATIC LITERATURE REVIEW (SLR) ……............................................. 9

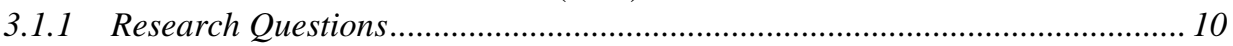

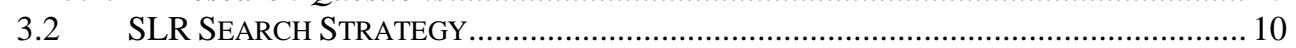

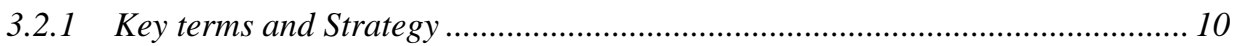

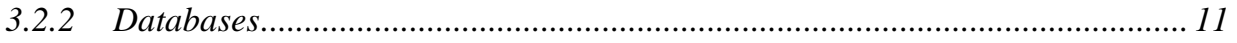

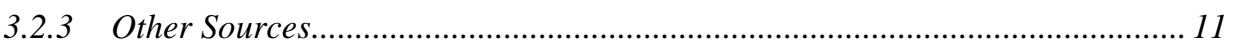

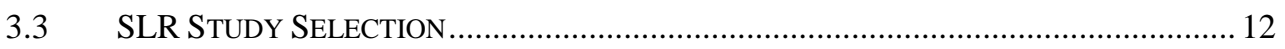

3.3.1 Study Selection Process........................................................................... 12

3.3.2 Study Selection Documentation................................................................. 13

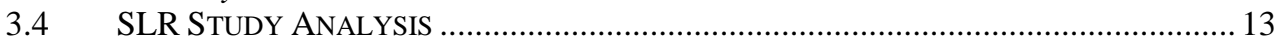

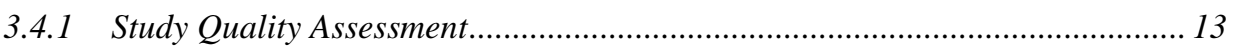

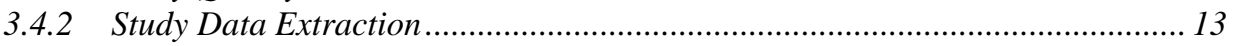

$3.5 \quad$ SLR STUDY ANALYSIS \& SYNTHESIS ............................................................... 13

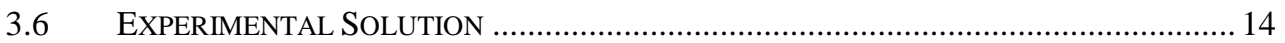

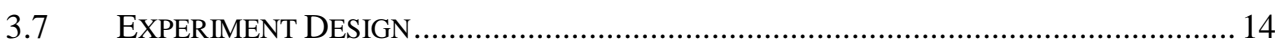

3.8 EXPERIMENT IMPLEMENTATION …………………........................................ 15

3.9 EXPERIMENT VALIDATION DESIGN ................................................................ 17

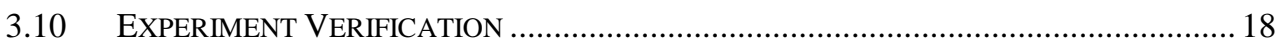

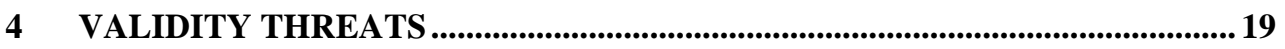

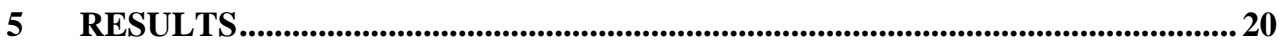

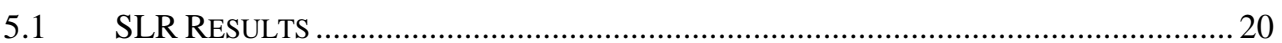

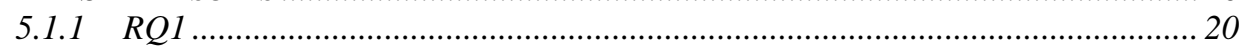

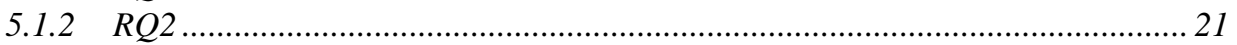

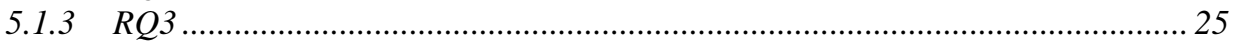

5.2 EXPERIMENT VALIDATION AND EVALUATION RESULTS …………………............ 26

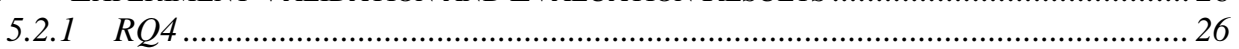

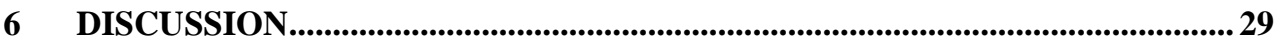

7 CONCLUSION AND FUTURE WORK ..............................................................

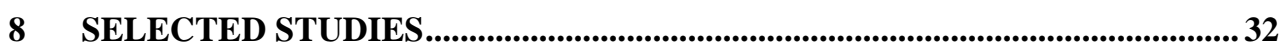

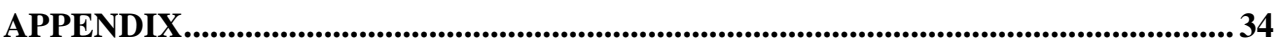

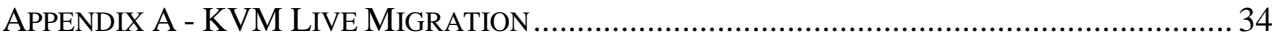

APPENDIX B - ReSEARCH METHODOLOGY ALTERNATIVE.................................................. 35

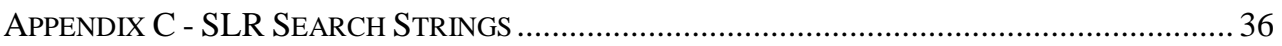

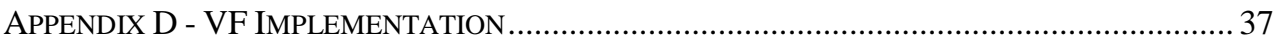

APPENDIX E - VF RULE VERIFICATION BEFORE MIGRATION ……………............................. 39

APPENDIX F - VF RULE VERIFICATION AFTER MIGRATION................................................... 40

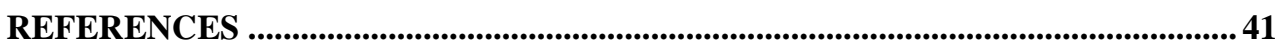




\section{List of Tables}

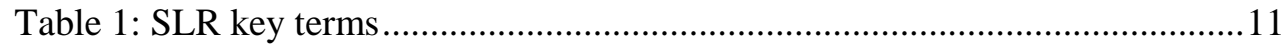

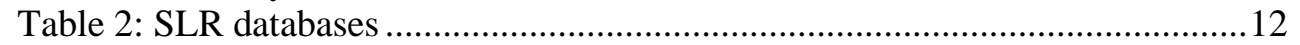

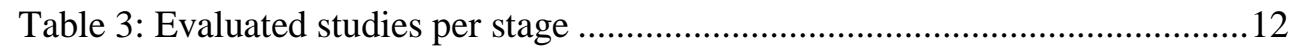

Table 4: Study quality assessment questionnaire ….............................................13

Table 5: Technical implementation specifications .................................................17

Table 6: Sources searched for all years (up to October 30 2012)...........................20

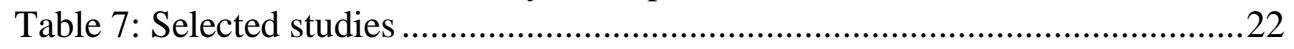

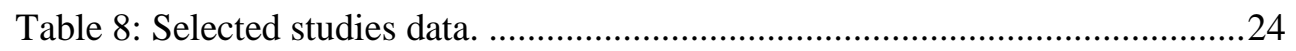

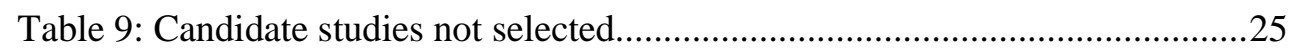

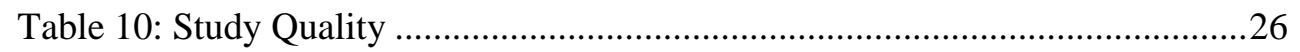

Table 11: Firewall intactness evaluation using ICMP packets...............................26

Table 12: Research methodology comparison ....................................................35

\section{List of Figures}

Figure 1: Role of VMM-level \& VM-level VF in VM migration............................2

Figure 2: System virtualization architecture.......................................................

Figure 3: VMM-level \& VM-level virtual firewall ................................................ 7

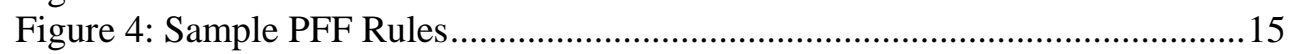

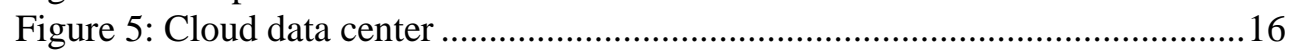

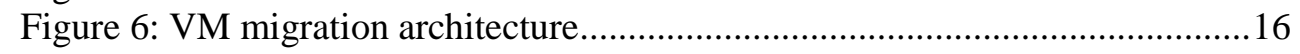

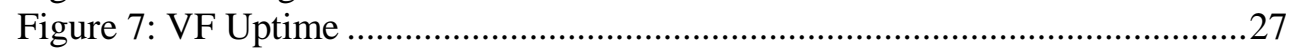

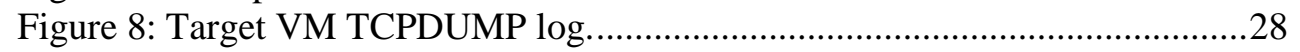

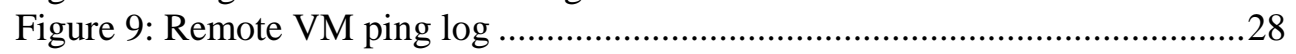

Figure 10: KVM live migration algorithm .........................................................3

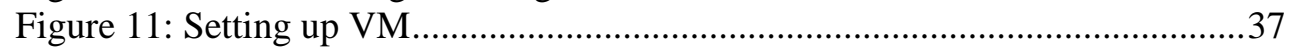

Figure 12: Remote VM firewall configuration......................................................

Figure 13: Target VM firewall configuration......................................................38

Figure 14: KVM Migration Interface .................................................................

Figure 15: PFF rule verification before migration. .............................................39

Figure 16: PFF rule verification before migration $(\log )$.........................................

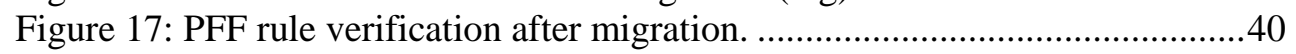

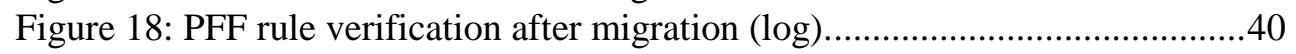




\section{INTRODUCTION}

CC changes the way IT architectural solutions are put forward using virtualization. The National Institute of Standards and Technology (NIST) ${ }^{1}$ defined CC as, model for enabling on-demand access to networks, storage, applications, and services (Mell and Grance, 2011). Examples of CC products are Amazon Web Service, Google AppEngine, and Windows Azure. Both small and large organizations benefit from CC in terms of reduced operating costs and increase in business agility (Garrison, 2012). The CC paradigm has attracted the attention of many academic and industrial researchers. European Network and Information Security Agency (ENISA) ${ }^{2}$ carried out a study in which CC security was labeled as an important research area (Catteddu and Hogben, 2009).

The key technique behind $\mathrm{CC}$ is virtualization. The inherent vulnerabilities of internal network in host and Local Area Network (LAN) pose threat to the VMs when they are live migrated. These security challenges are presented in (Modi et al. 2012; Qina et al. 2012; Ranjith et al. 2012; Dawoud et al. 2010). It is believed that the VM migration security is an important research area.

Live VM migration is a powerful feature of virtualization. In it the VMM triggers the migration and VM is moved from one physical host to another with little or no delay and without service interruptions (Xianqin et al., 2009). VM migration allows the CPs to do upgrades, workload balancing, consolidation of VMs, testing and etc.

In the Cloud data center, multiple VMs (with guest OS) run concurrently on a physical computer (host) using VMM. The network inside host and the LAN, in which migration is done, is vulnerable to attack just like physical network. This puts the VM guest OS and the applications hosted on it at risk (Modi et al., 2012; Tavakoli et. al, 2012; Wu and Winer, 2010). The VM faces vulnerabilities when it migrates from one host to another (Clark et. al, 2005; Oberheide et. al, 2008; Shetty et. al, 2012). VM live migration is susceptible to network attacks such as ARP spoofing, Man-in-the-middle, DoS, DDoS, DNS poisoning and etc. (Oberheide et al. 2008; Modi et al. 2012). As different customers run VMs in same LAN (in which the migration is done) the VM becomes prone to attacks (Tavakoli et al. 2012). An inappropriate firewall policy allows an attacker to initiate, control and terminate the migration (Shetty et al. 2012; Dawoud et al. 2010). A weak or missing policy can help an attacker to fake migration, insert VM with malicious code, execute rouge code on it or crash it. To prevent any unauthorized activity the control policy such as firewall rules must be defined and kept intact all times during migration process (Shetty et al. 2012; Dawoud et al. 2010).

Hardware firewalls are blind to virtual traffic since it never leaves the host. Hence, the virtual firewalls (VF) are used to secure VMs. VMM-level VF are discussed and implemented by (Basak et. al, 2010; Carlin and Curran, 2011; Garber, 2012; Sqalli et. al, 2011). Source VMM-level VF provides security to VM before the migration incurs and the destination VMM-level VF starts securing VM after migration is completed. It thus, becomes possible for attacker to use the intermediate migrating window to launch attacks.

Considering the potential of VFs there should be a great value in using open source VFs at VM-level for protecting VMs during migration (Basak et al. 2010). The

\footnotetext{
${ }^{1}$ http://www.nist.gov

${ }^{2}$ www.enisa.europa.eu
} 
open source community has developed firewall solutions such as OpenBSD Packet Filter firewall, FreeBSD PFSense, and etc. OpenBSD is most mature OS and has more security focus than other Linux flavors. It has only become stable and richer in security features over the years (Artymiak 2003). OpenBSD PFF has been ported to other OSs. Its features ${ }^{3}$ include stateful filtering, IPv4 and IPv6 filtering, packet normalization, logging, dynamic rule-sets, bandwidth shaping, load balancing, spam filtering and etc. with huge support community. Hence, for this study OpenBSD PFF is implemented on VM.

Having VF on machine would provide security to VM after migration is initiated to when the migration ends, thus, protecting VM from attacks during the transition. With PFF at VM-level the attack window for attacker during migration span can be reduced. The results of this study can be useful for the Cloud providers (CPs) and customers. Having VM-level firewall would build security layer for customer. If all customers have this layer, the security of overall data center would also be enhanced, thus, benefitting $\mathrm{CP}$ in the long run.

\subsection{Problem Definition}

In $\mathrm{CC}$ the virtualization technology leverages running many VMs on a single physical host. The VMs are live migrated to other hosts by CPs for load balancing, maintenance, and etc. VM is exploitable during migration. During migration the intruder in network can stop migration, manipulate data on VM or hijack it (Oberheide et al. 2008; Modi et al. 2012; Dawoud et al. 2010). If an intruder is successful to gain access to VM during transition, the OS and data on VM is exposed. This propagates the threat to its host and other hosts in LAN. VFs, which are regarded as first line of security in network, are used to secure the VMs when they migrate (Basak et al. 2010; Cabuk et al. 2008). These protect the VM from virtual network and LAN threats using firewall rules to control the VM communication. VFs can be at VMM-level or VMlevel. The VMM-level VFs, if employed, protects VM before and after migration. Different VF solutions are available in industry that takes care of VM migration security at VMM-level.

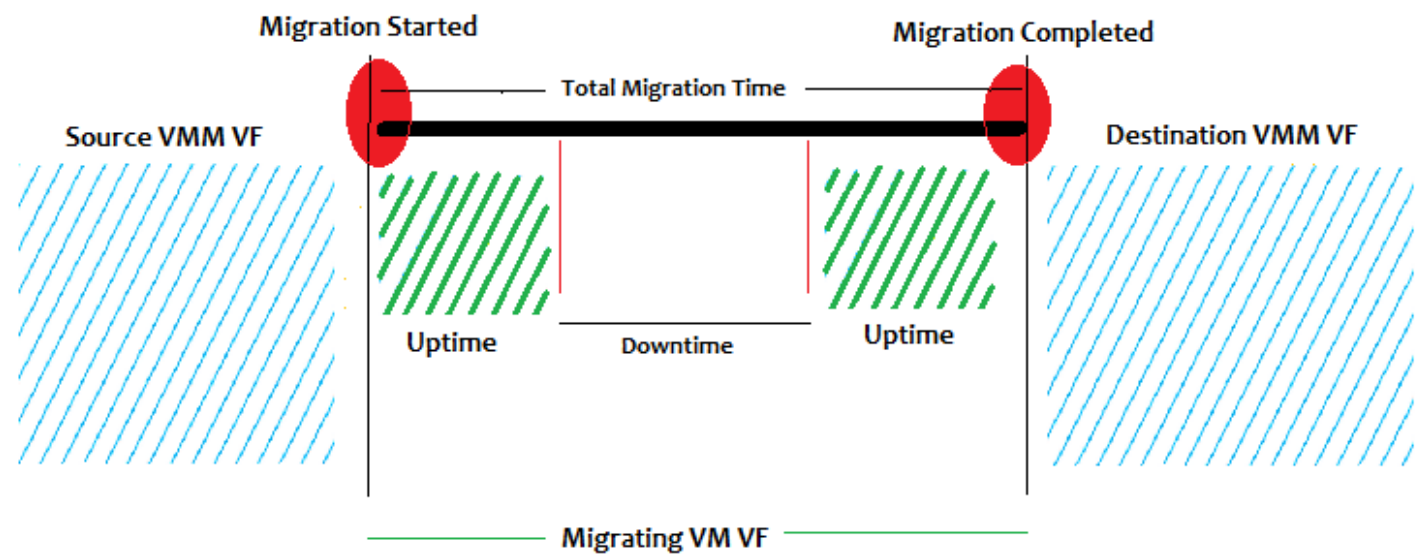

Figure 1: Role of VMM-level \& VM-level VF in VM migration

To compare the VMM-level and VM-level VFs for VM migration security, Figure 1 can be referred. The VMM-level firewalls protect the VM from network threats before and after migration (Dawoud et al. 2010; Shetty et al. 2012). During the

\footnotetext{
${ }^{3}$ http://www.openbsd.org/faq/pf/filter.html
} 
migration the attacker anywhere in network can exploit the VM (Oberheide et al. 2008; Modi et al. 2012). The VM-level firewall would enable security of VM during migration. To evaluate the intactness of VM-level VF the Total Migration Time (TMT) and VM downtime (DT) is calculated. The difference of both would indicate the uptime (UT) of VM-level VF during VM mobility.

TMT is the duration between when migration is initiated and when the migration is completed (Clark et al. 2005). TMT ranges between $21 \mathrm{~s}$ to $250 \mathrm{~s}$ (Xianqin et al. 2009; Salfner et al. 2011). The DT during migration is when the VM is unresponsive. VM downtime varies between $0.3 \mathrm{~s}$ to $14 \mathrm{~s}$ (Xianqin et al. 2009; Salfner et al. 2011). DT is a slot anywhere during TMT and according to literature its always less than TMT (Salfner et al. 2011). Hence, the time during which migration is happening but $\mathrm{VM}$ is not down could potentially be used by attacker to attack VM, which is where the VF can provide security to VM. This time is referred as VF uptime (UT). It is calculated by subtracting DT from TMT. Hence, UT for VF is calculated by sending ICMP packets continuously to the migrating VM. The machine has firewall policy that allows the ICMP packet transmission. The firewall log files are analyzed to calculate TMT and DT (Salfner et al. 2011; Hao et al. 2009). UT for VF is measured as no other metric to measure uptime for VF is exposed by VF vendor or Cloud provider (Salfner et al. 2011).

Having VMM-VF can have other associated issues. The VMM is a juicy target for attackers. It is also known as attack surface. VMM triggers the migration instruction to which the customer is unaware. These add risk to the software and OS residing on VM during migration and until the firewall rules are restored. The activeness of VF during migration is not researched. This study performs its implementation and evaluates the UT of VF. Consequently, having the VM-level VF intact during the migration with VMM-level firewall would enable additional and customized VM migration security.

\section{$1.2 \quad$ Research Questions}

Having known the context of VFs in VM migration in CC the research questions are:

RQ1. How much research is published on virtual firewalling for VM migration security in $\mathrm{CC}$ ?

RQ2. What are the techniques addressed for virtual firewalling for VM migration security in CC?

RQ3. Are the researches limited?

RQ4. Does VF at VM-level protect the VM during migration and what is its empirical evaluation?

\subsection{Aims and Objectives}

The aim is to investigate the VM-level virtual firewalling solution as a complementary security layer for securing the migrating VM in CC domain. The objectives are as follows:

- Identify how VFs secure the migrating VM in CC and its research gaps.

- Propose virtual firewalling at VM-level using open source VF.

- Implement VM-level VF to validate its intactness (uptime) during migration.

- Validate and evaluate the VM-level VF in real Cloud data center environment.

\subsection{Contribution}

This research contributes towards identifying literature and research gaps on virtual firewalling for migrating VMs in $\mathrm{CC}$. Moreover, it provides understanding of open source virtual firewalling at VM-level for migrating VMs to reduce attack 
window of VM during the migration. The final contribution is the validation and uptime evaluation of the implemented Packet Filter firewall for VM at VM-level during migration in City Network data center.

\subsection{Thesis Structure}

The following sections are organized as follows: Section 2 comprises of the background and related work on virtual firewalls for VM migration security. Section 3 describes the research methodology i.e., the approach followed to undertake SLR and to conduct the experiment. Section 4 presents the validity threats. Section 5 shows results of SLR and experiment along with experiment validation. Section 6 contains the discussion on the gathered results. Section 7 finishes off the research with concluding remarks and potential pointers for future work. Section 8 lists all selected studies for SLR.

\subsection{Acronyms}

$\begin{array}{ll}\text { CC } & \text { Cloud Computing } \\ \text { CP } & \text { Cloud Provider } \\ \text { ICMP } & \text { Internet Control Message Protocol } \\ \text { LAN } & \text { Local Area Network } \\ \text { OS } & \text { Operating System } \\ \text { PFF } & \text { Packet Filter Firewall } \\ \text { SLR } & \text { Systematic Literature Review } \\ \text { VF } & \text { Virtual Firewall } \\ \text { VM } & \text { Virtual Machine } \\ \text { VMM } & \text { Virtual Machine Monitor } \\ \text { WAN } & \text { Wide Area Network }\end{array}$




\section{BACKGROUND}

Virtualization is a staple technology of CC. IBM pioneered it in 1960s (Armbrust et al., 2009) to utilize the resources of mainframe computers with timesharing and multiprogramming techniques. These techniques led to the conception of virtualization. Virtualization is defined as the abstraction of hardware resources for enabling resource sharing. It can be at different levels: application, desktop, network, storage and system virtualization (Qina et al. 2012). These resources can be dynamically provisioned to the users on demand via Internet. With virtualization benefits comes the need for virtual security (Bellovin 2006; Qina et al. 2012; Garber 2012).

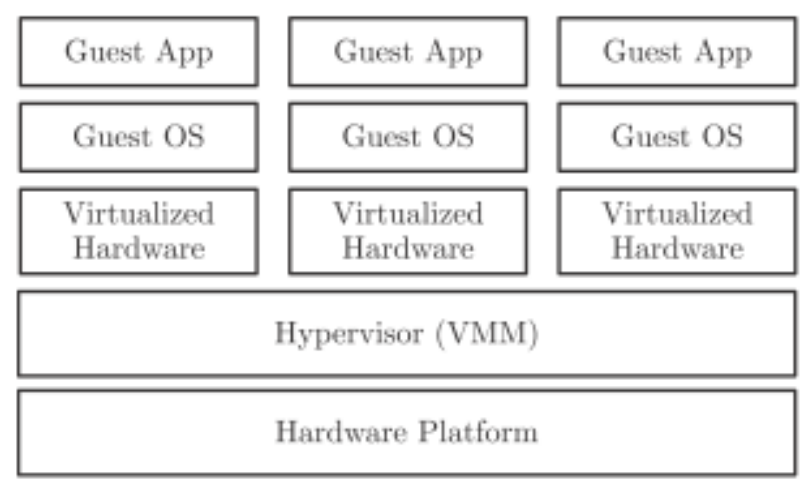

Figure 2: System virtualization architecture

System virtualization is when a single physical host runs a number of VMs on it. This VM has its own applications that run on its OS (guest OS). For the user, a VM behaves just like an independent physical machine. A Virtual Machine Monitor (VMM) interconnects the VMs, manages them and emulates the underlying hardware for VM (Qina et al. 2012). Examples of VMMs are VMware ESXi ${ }^{4}$ and $\mathrm{KVM}^{5}$. For emulation, environment such as QEMU can be used. The system virtualization architecture (taken from (Qina et al. 2012)) is shown in Figure 2.

Live VM migration is a powerful feature of virtualization. In it the VMM triggers the migration and VM is moved from one physical host to another with little or no delay and without service interruptions (Xianqin et al., 2009). VM migration allows the CPs to do upgrades, workload balancing, consolidation of VMs, testing and etc.

VM live migration is susceptible to network attacks such as ARP spoofing, Manin-the-middle, DoS, DDoS, DNS poisoning and etc. (Oberheide et al. 2008; Modi et al. 2012). As different customers run VMs in same LAN (in which the migration is done) the VM becomes prone to attacks (Tavakoli et al. 2012). An inappropriate firewall policy allows an attacker to initiate, control and terminate the migration (Shetty et al. 2012). A weak or missing policy can help an attacker to initiate migration, insert VM with malicious code, execute rouge code on it or crash it. To prevent any unauthorized activity the control policy such as firewall rules must be defined and kept intact all times during migration process (Shetty et al. 2012).

\footnotetext{
${ }^{4}$ http://www.vmware.com

${ }^{5} \mathrm{http}: / /$ www.linux-kvm.org
} 
The authors' state firewalls can enforce security for VM in network (Basak et al. 2010; Dawoud et al. 2010; Tavakoli et al. 2012). They emphasize on firewall intactness during and right after migration to avoid any security loopholes. Computer firewalls have existed since 1980s (Ingham \& Forrest 2002). A firewall is a security device which enforces security policy using a set of rules. It can be virtual or physical. According to (Garber 2012) housing multiple VMs into one host eliminates physical/hardware firewall and other traditional security mechanisms. Author explains having virtualization adds a virtual layer in the IT infrastructure in which traditional hardware firewall, intended for physical environments, lacks visibility which adds up to the network vulnerabilities. CEO of Cisco, John Chambers, in 2009 RSA conference highlighted the point that security in Cloud cannot be handled in traditional ways (Greene 2009). Hardware firewalls cannot filter the virtual traffic as the virtual network traffic remains inside the host (Basak et al. 2010). As the Cloud environment is shared, it is possible for an attacker to launch an attack across VMs inside host or in LAN (Li, 2010; Modi et al., 2012). In a virtual world the attacks can be more rapid and thus, devastating (Kourai et. al, 2012; Sqalli et al., 2011).

VFs are a solution to this problem (Basak et al., 2010; Haletky, 2008; Modi et al., 2012; Northcutt, 2009; Zaborovsky et al., 2011). Virtual firewalling is a firewall service that can perform packet filtering in virtual environments based on IP, ports and protocols (Scarfone \& Hoffman 2009). VF policy for controlled communication of VMs can protect VM during migration from unauthorized migration initiation, hijacking, or inserting code into VM (Shetty et al. 2012).

The use of VF at VMM-level is discussed in the literature (Carlin and Curran 2011; Basak et al. 2010; Cisco 2011; Modi et al. 2012). Amazon EC2's firewall solution is discussed in Carlin and Curran's study. Basak wrote about VMware implemented vShield firewall which is a VM with hardened firewall capabilities. It is used to secure VM migration security in coordinating with VMware vMotion protocol. Cisco Virtual Security Gateway is also a VF that provides security policies on the virtual layer in CC.

Having VMM-level VF can have issues. According to (Szefer et al., 2011), the VMM is the attack surface and must have bare minimum control. There are more than 40 combined vulnerabilities reported in Xen and VMware ESXi in the U.S. government's National Vulnerabilities Database ${ }^{6}$. Work on secure VMM to perform secure migration has also been published (Xianqin et al. 2009). In addition to this, there could be an unsecure destination VMM or bugs in VMM migration module (Shetty et al. 2012). Jennifer Rexford suggests involving customer in the migration process as a good practice (Szefer et al. 2011). Mostly, the CP initiates migration to which the customer is unaware. Moreover, the source VMM-level VF protects VM before migration initiates whereas destination VMM-level VF protects VM after VM migration is under the control of destination host. The intermediate migration path remains unprotected making the VM vulnerable. This is demonstrated in Figure 3.

\footnotetext{
${ }^{6}$ http://nvd.nist.gov
} 


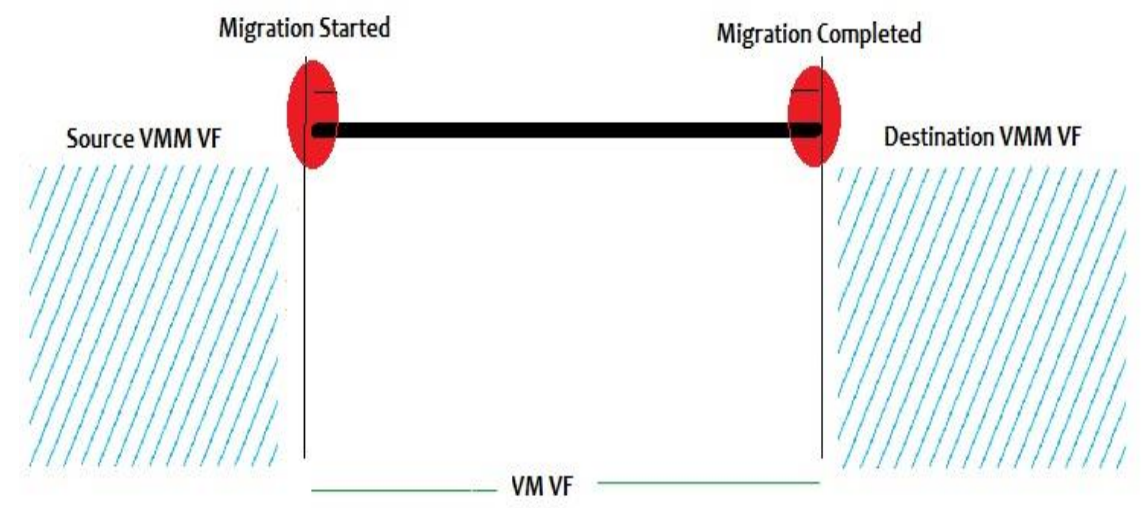

Figure 3: VMM-level \& VM-level virtual firewall

Considering the potential of VFs there should be a great value in using VM-level open source VFs for protecting VMs between start migration and end migration (as in diagram). Most of the previous work has focused on the implementation of live migration with little or no consideration towards its security intactness while migration. Ones that discuss security mainly cover aspects of VMM-level firewalls. The intactness or activeness of VM-level VF during migration or its empirical evaluation was not researched. VF is considered an important integrated security element in Cloud environments. It is, however, good to secure VM using VM-level firewall i.e. at user level to provide security to VM during migration. This would also wave-off the total dependency on VMM-level VFs and reduce other associated risks. An accessible solution for this is OpenBSD PFF. In recent years open source VFs have matured and are effective like their commercial counterparts (Patton et al. 2000; Cabido 2011; Ryan 2002). PFF amongst other open source VFs provides richer security features (Artymiak 2003). Hence, it would be beneficial for migrating VM's security to implement VM-level firewalling. It would be advantageous to experiment and validate the use of PFF at VM-level for migrating VM as a complement to enhance its security during VM transition. In this, study Kernel-based VMM (KVM) is used to perform migration. KVM live migration algorithm ${ }^{7}$ is appended in Appendix A.

\subsection{Related Work}

A closely related literature to this study is (Basak et al. 2010), in which the authors present the need for virtualizing security functions that are currently in the form of hardware appliances, such as firewalls. They demonstrated that VMware vShield VF secures VM and helps to achieve secure migration with coordination of vMotion protocol. For secure migration the vShield VF should be installed on every VM and the vShield manager should be available at all times. The vSheild manager would send firewall rules to every vShield VF before executing migration to maintain protection. It runs on the commercial security vulnerability appliance of VMware only. A design approach and prototype implementation of an extension of the virtual network (ViNe) system is presented in (Tsugawa, 2010) to support live migration of VMs. ViNe established tunnels between Cloud infrastructures which doesn't interrupt the network traffic. The researcher executed migrations in which both the source and destination VMMs should have ViNe to support the migration process. Moreover, the evaluation of the approach was not presented.

\footnotetext{
${ }^{7}$ http://www.linux-kvm.org/page/Migration\#Algorithm_.28the_short_version.29
} 
(Miaol et al., 2010) imitated network device called VSFilter which performed virtual firewalling to filter network packets. However, it did not talk about VF behavior during migration. A network virtualization solution is presented in (Xu et. al, 2011) that provided WAN VM live migration without disturbing the network connections. They do not specifically talk about firewall rules. (Wu \& Winer 2010) recommended isolating the network traffic between VMs housed on same host using a VMM-based firewall. Other literature on VMM-level firewall include (Carlin and Curran, 2011; Cisco, 2011; Modi et al., 2012).

The above mentioned literature advocated use of a VMM-based firewall for securing a VM during migration. A related work that acknowledges VM-level security is given in (Tavakoli et al. 2012). The authors introduce a framework for moving firewall context of VM with VM. They base the migration for LAN environments and using shared file system like in this study. However, they neither evaluate their work nor refer to firewall rule behavior during migration. (Xianqin et al. 2009) also acknowledged this issue of migrating firewall states, and modified the XEN live migration tool for providing connection tracking at VMM-level. However, none implemented VF at VM-level, nor discussed its intactness during migration and neither performed empirical tests for VF resiliency during VM motion. 


\section{RESEARCH METHODOLOGY}

Collecting data using diverse methods provides a better understanding of a research problem (Creswell 2009). For this reason the research questions proposed for this dissertation are answered using mixed method approach. The study begins with a SLR for the knowledge on virtual firewalling for VM migration security. In second phase, open source VF is implemented on VM to identify potential of having VF on VM during migration. This is done by measuring firewall behavior or intactness during migration. As it's not possible to test firewall rule during small migration time, hence, the uptime (UT) of VF is calculated. UT denotes that firewall is active and is providing security to VM during migration span. An experimental validation in real Cloud setup is performed to evaluate the VF intactness during migration.

Initially, interview, was chosen as a data collection method instead of SLR, assuming first-hand knowledge and experience can answer well the research questions about using VFs to secure VM migration. This methodology was dropped in the initial stage due to limited number of data centers within reach, and instead SLR was chosen to maximize the data collection. Appendix B compares both methodologies with respect to this study.

The systematic review helped to gain the knowledge presented in literature regarding virtual firewalling for migrating $\mathrm{VM}$ and in identifying its research challenges. After analyzing the available literature and limitations the experimental solution was designed, validated and evaluated. To design the experiment the available resources were taken into consideration which included the accessible resources at the City Network. In the next step the VF was configured on VM to verify the VF rules before, during and after migration. A pilot migration was performed to verify rule behavior during migration. To understand to what extent the experimental results are valid, the migration expert at City Network performed 8 VM migrations. However, firewall evaluation is critical in case of live VM migrations, hence, the UT is calculated. It is measured using difference of TMT and DT (Hao et. al, 2009). The difference in both durations shows the potential of VF in securing VM from network attacks during migration. The following sections describe these steps in detail.

\subsection{Systematic Literature Review (SLR)}

IT practitioners and decision makers decide about the technological aspects of the organization. It is easy for them to take informed decisions about the technology if there is enough evidence about its limits, risks, qualities, costs and suitability. (Barbara 2007) suggests use of evidence-based computing to support and improve the technology adoption decisions. The goal of evidence-based research in computing discipline is to integrate current best evidence with practical experience to enable decision making about the development and maintenance of technology.

In this context, evidence is the synthesis of quality scientific studies on a particular topic. The main method of synthesis is a SLR (Barbara 2007). SLR is a methodological in-depth review of research results to support the development of evidence-based guidelines to provide appropriate technological solutions in a specific context. It is a predefined and fair process making it repeatable and less biased as compared to other reviews, such as meta-analysis (Barbara 2007). The choice of SLR, among other review methods, is also based on author's familiarity with the method. 
The purpose of SLR in this study is to review the how VFs, secure VM during migration in CC domain using primary studies (so this study is a form of secondary study). It is important to look into the topic as discussed in above sections.

Before undertaking the SLR, it was queried that no similar work is done. Following databases were searched; Engineering Village, IEEE Xplore and ACM Digital. The following keywords and combination of Boolean operators were used to query: ("virtual machine*" OR "virtual Machine*" OR "virtual Network*" OR "virtual machine monitor" OR "hypervisor*") and (firewall* OR Firewall*) and (migrat* OR mobility OR transfer*) and (SLR OR "Systematic Literature Review OR "Systematic Review" OR "literature review" OR "literary review"). There were no related results retrieved.

The SLR was conducted based on the guidelines of (Barbara 2007). The protocol for addressing the research objectives proposed in Section 1.3 is presented in the following subsections. The search strategy is described in Section 3.2, selection methodology in Section 3.3, analysis in Section 3.4 and study synthesis in Section 3.5. In Section 5.1 the SLR related research questions (RQ1-RQ3) are answered.

\subsubsection{Research Questions}

The research questions are given in Section 1.2.

With respect to RQ1, the research was conducted without applying any start year to collect all the relevant published literature. To address RQ1 following were identified; number of papers published per year, the databases and journal/conference of paper. With respect to RQ2, the underlying virtual firewalling techniques for VM were identified. To answer RQ3 2 issues are looked into:

RQ3.1. Are the research topics limited?

RQ3.2. What is the quality of literature?

\subsection{SLR Search Strategy}

This section outlines the process for generating key terms, the strategy for searching, the databases searched, and the documentation for the search.

\subsubsection{Key terms and Strategy}

The key terms were identified for the literature search from prior knowledge in the subject area and by consulting Sogand Shirinbab, a PhD. student in Blekinge Tekniska Högskola (BTH). Her research is about VM migration in CC with focus on performance. However, key terms related to 'migration', 'virtual machine' and 'Cloud computing' were common in both. The key terms are "virtual machine", "migration" and "firewall". To define key terms, Population, Intervention, Comparison and Outcome (PICO), method as suggested in (Barbara 2007) was followed. Key term 'Cloud Computing' was chosen as population, 'Virtual Machine', 'Migration' and 'Firewall 'as an intervention. No comparison and outcome criteria were specified.

As identifying primary studies can be difficult (even with knowledge in area) it is a good approach to evaluate search strategy to verify if it is capable of returning most relevant matches. For this Quasi-gold standard evaluation technique was used $(\mathrm{H}$. Zhang et al. 2011). Sensitivity of strategy for a given topic is defined as the proportion of relevant studies retrieved for that topic ${ }^{8}$. High sensitivity is usually desirable. The sensitivity for the search strategy used in this SLR is $(26 / 36 * 100)$ i.e. $72.2 \%$. This sensitivity is acceptable according to scale in (H. Zhang et al. 2011).

\footnotetext{
${ }^{8}$ Sensitivity $=($ Number of selected studies retrieved $/$ Total number of relevant studies $) * 100 \%$
} 
To perform search on electronic library the key terms (with synonyms and variations of spellings) as in Table 1 were defined.

Table 1: SLR key terms

\begin{tabular}{|l|l|l|l|}
\hline 1: Virtual Machine & 2: Migration & 3: Firewall & 4: Cloud Computing \\
\hline Virtual machine & Migrate & Firewall & Cloud \\
\hline Virtual machines & Migrates & Firewalls & Clouds \\
\hline VM & Migration & Firewalling & \\
\hline Virtual network & Transfer & & \\
\hline Virtual networks & Transferring & & \\
\hline VN & Transfers & & \\
\hline Virtual machine monitor & Mobility & & \\
\hline VMM & & & \\
\hline Hypervisor & & & \\
\hline Hypervisors & & & \\
\hline
\end{tabular}

Database search strings combined the key terms from all 4 columns in Table 1 . Using each combination from all columns, there were 420 search strings for each database. In all databases the advanced search feature was used and search terms were entered as one string. First column terms were entered with OR operator in between all terms. Likewise terms of column 2, 3 and 4 were added. All column terms were combined with the AND operator. The key terms were used with wildcards, initial capital letters, and field preferences in scientific databases. Papers were reviewed if they had the key term (column 1 and 2 of Table 1) in the abstract. No field was specified for last 2 columns. The search strings are in Appendix C.

\subsubsection{Databases}

Nine scientific literature databases were searched for the literature. The selected databases are shown in Table 2. The choice of these sources are based on the database recommendations given in (Barbara 2007) ${ }^{9}$ and in Structured Literature Review ${ }^{10}$ in Computer Science published by senior research scientist at SINTEF ${ }^{11}$ (Kofod-petersen 2012). Also the search strategy evaluation using Quasi-gold sensitivity was done before proceeding (H. Zhang et al. 2011).

When databases allowed advanced search options, non-refereed papers such as news articles, and books were excluded. Additionally, search was restricted by Computer Science in Springer Link as it included a lot of non-referred work. The journals and conference papers were selected because journals are peer-reviewed and also because they are known to include empirical studies which are considered primary for this SLR, and, conference and workshop papers have practical and novel insights on any research area (e.g.: (Szefer et al. 2011)).

\subsubsection{Other Sources}

In addition to this, Sogand Shirinbab, a PhD. student at BTH was directly contacted to ask for any sources or unpublished literature. It was done because according to (Barbara 2007) other sources of evidence must also be searched to make the full literature review. Moreover, the reference lists of relevant studies were skimmed. This added 4 papers to final set of selected studies (Wu \& Winer 2010; Miaol et al. 2010; Dawoud et al. 2010; Xianqin et al. 2009).

\footnotetext{
${ }^{9}$ IEEExplore, ACM Digital library, CiteSeer, Google scholar, Inspec, ScienceDirect, EI Compendex, SCOPUS, SpringerLink.

${ }^{10}$ ACM digital library, IEEE Xplore, CiteSeer, ISI web of knowledge, ScienceDirect, SpringerLink,

Wiley Inter science.

${ }^{11} \mathrm{http}: / /$ www.sintef.no
} 
Table 2: SLR databases

\begin{tabular}{|l|l|}
\hline No. & Source \\
\hline 1 & ACM Digital library \\
\hline 2 & CiteSeer \\
\hline 3 & Engineering Village (Inspec, Compendex) \\
\hline 4 & IEEExplore \\
\hline 5 & ISI Web of science \\
\hline 6 & ScienceDirect \\
\hline 7 & SCOPUS \\
\hline 8 & SpringerLink \\
\hline 9 & Wiley Inter science \\
\hline
\end{tabular}

\subsection{SLR Study Selection}

This section describes the process and documentation used for selecting studies for the SLR.

\subsubsection{Study Selection Process}

Selection of studies for inclusion in the SLR is a two-stage process: (1) initial selection of studies was based upon key terms search in the abstracts; in electronic databases where the advanced search feature for abstract was not possible (e.g. CiteSeer, SpringerLink) reading of abstract was done manually from the queried results; only results having key terms (column 1 and 2 of Table 1) in abstract were forwarded to next stage (2) First the duplicated papers were excluded and further selection of studies was done based upon reading the paper. Table 3 shows the number of papers evaluated at each stage of the selection process.

Table 3: Evaluated studies per stage

\begin{tabular}{|l|c|c|c|c|}
\hline Stage & $\begin{array}{c}\text { Papers at start of } \\
\text { Stage }\end{array}$ & Added papers & Total papers & Selected papers \\
\hline Stage 1 & All & 0 & All & 36 \\
\hline Stage 2 & 36 & 0 & 36 & 12 \\
\hline Final: selected studies & 12 & 6 & 18 & 18 \\
\hline
\end{tabular}

At Stage 1, all 9 databases were queried with the key terms. There were 36 papers that had relevant abstracts and warranted further reading in Stage 2. In Stage 2, the duplicates were removed. In the start of the final stage there were 12 selected studies. Latter 6 studies were added. Using reference list of selected papers 4 papers were retrieved, (Wu \& Winer 2010; Miaol et al. 2010; Dawoud et al. 2010; Xianqin et al. 2009). A search was redone for a conference proceeding keynote (Rexford 2012) using the name of speaker for any other related paper on the subject. This resulted in adding (Szefer et al. 2011). Similarly, (Riteau 2011) manuscript was replaced with author's conference paper (Tsugawa et al. 2010). These were added to final selected studies.

Inclusion and exclusion criteria were focused on identifying papers that mention about virtual firewall for VM migration specifically in CC. The inclusion and exclusion criteria helped to refine the search to meet the research objectives.

All articles on the following topics published till October 30th 2012 was included:

- Virtual firewalling covered for VM migration security.

- All empirical and theoretical papers were included.

- Papers published in workshop, conference or journal.

Articles excluded in the selection process met the exclusion criteria as below:

- Duplicate reports of the same study (when several reports of a study exist in different sources the latest version of the study was included).

- Studies not in English. 
- Papers where main topic is not securing VM migration using VFs in CC.

\subsubsection{Study Selection Documentation}

The study data were stored in MS Excel for every stage of the selection process.

For each study, some or all of the following information was maintained:

- Title

- Source type and source

- Database

- Reference and publication year

Additionally, for Stage 2 in the study selection, the reason for exclusion was recorded. After a stage was complete, the selected studies moved to another column in Excel file, and any additional information required for selection was obtained.

\subsection{SLR Study Analysis}

After all stages of study selection were complete, the quality of the selected studies was assessed and data was extracted from studies. Quality assessment was done to determine the strength of elaborated inferences. It was done to ensure relevant literature is considered during the process (Barbara 2007). The following sections describe the data collected from each of the selected studies.

\subsubsection{Study Quality Assessment}

The quality of all of the selected (final stage) studies was done. For each study the questions outlined in Table 4 were answered. All of the questions have 3 possible responses: yes (1), no (0), or somewhat (0.5); where number is the numerical value for each response. The sum of responses for each quality assessment question gives a relative measure of study quality. The choice of questions was based on quality checklist guidelines of (Barbara 2007). Table 4 shows the study quality assessment.

Table 4: Study quality assessment questionnaire

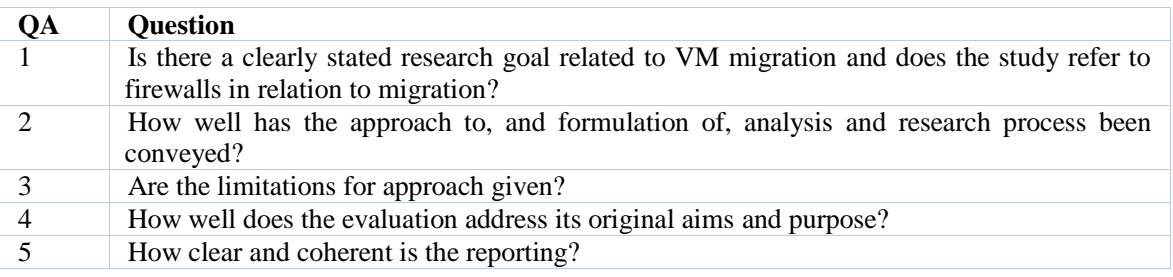

\subsubsection{Study Data Extraction}

The data from the selected studies were maintained in paper notes and Excel file.

For each selected study following additional data was extracted:

- Firewall role in VM migration

- LAN or WAN Migration

- VM or VMM level security

- Approach limitations

- VF evaluation

- VMM used in implementation

- Summary of the study

\subsection{SLR Study Analysis \& Synthesis}

For each research question, data was collected, analyzed and synthesized for each selected study. For analysis data was collected from papers based on research questions. Web diagrams were created from the extracted data (Christine B. Feak 2009). The diagrams were refined based on the theme of question. The irrelevant 
extracted data was removed and final set of data was tabulated. Table 6 and Table 7 provide number of papers published per year and their source databases addressing RQ1. Section 5.1.2 provides answer to RQ2 by providing high-level overview on VF used for VM migration. Table 8 presents data in tabular form for RQ2. Section 5.1.2 and 5.1.3 describe the results for VF techniques and gaps in literature concluding RQ2 and RQ3.

\subsection{Experimental Solution}

Based on the results of RQ2 and RQ3 the experimental solution is designed, implemented and validated which answers RQ4. RQ4 looks like this; does VF at VMlevel protect the VM during migration and what is its empirical evaluation?

The SLR helped to gain the knowledge presented in literature regarding virtual firewalling for migrating VM and in identifying its research challenges. The SLR showed trend of VMM-level firewall for VM migration security with no research on firewall behavior during migration. Neither was VM-level firewall solution tested and evaluated. After analyzing the gaps the experimental solution of having VM-level VF for VM migration was put forward and its intactness calculated using uptime to find out the potential of having VM-level VF over VMM-level VF for VM migration security. Experimental design, implementation, verification and validation are discussed in the following sections.

\subsection{Experiment Design}

Before using VM-level PFF in real Cloud environment, it is important to investigate whether the VF rules remain intact during migration or not. In addition to this the uptime of VF during migration is evaluated to estimate the potential of using VM-level VF. The uptime (UT) is calculated using the difference of Total Migration Time (TMT) and downtime (DT). UT = TMT $-\mathrm{D}$.

TMT is the duration from when the migration begins to when the migration is completed. DT is defined as the phase during migration when there is VF unreachability (Salfner et al. 2011). Both TMT and DT are critical metrics for evaluating VM migration. These are used in the experiment to evaluate the intactness of VF during migration as no other metric to evaluate VF behavior during migration is provided by $\mathrm{CP}$, who conducts migration, nor its given by VF vendor (Salfner et al. 2011).

Both TMT and DT are measured using ping commands. Ping is used here as virtualization products do not expose TMT and DT themselves (Salfner et al. 2011). Similar approach was used in Fang Hao's study to estimate DT (Hao et al. 2009). The ping requests were directed to the target VM from remote VM after every 0.5 second and 0.01 second intervals (I). The choice of decreasing the interval was to get more precise results. To find out TMT 2 different formulas we used for different ping intervals. For $\mathrm{I}=0.5 \mathrm{~s}$, TMT $=\mathrm{PS} / 2$ and for $\mathrm{I}=0.01 \mathrm{~s}$, TMT $=\mathrm{PS} / 100$; where, PS is total number of ping packets sent by remote VM from start of migration till when the migration was completed and $\mathrm{I}$ is the ping interval. The formula for calculating downtime is: $D T=P L^{*} I$; where, PL denotes the number of lost pings and $\mathrm{I}$ is the ping interval (Salfner et al. 2011).

To validate the successful VM migration the pre-migration and post-migration VF configuration files are recorded and compared. This comparison was done manually. Then rules were tested before and after migration to verify their functionality. To test the intactness of firewall rules an ICMP echo rule (called ping) is tested during migration. The firewall intactness is evaluated in terms of VF uptime (UT). 
In order to achieve the general results in experiments they should be conducted in real environments with actual people who are going to benefit from it or use it (Wohlin, 2000). However, as VMs are owned by customer, hence, the experiment was conducted in real setup by renting VMs from City Network. The diagrammatic representation of the setup is in Section 3.8. However, the intervention of migration expert at City Network was done to perform the migration.

To design the experiment the population is to be identified (Wohlin, 2000). The population consists of PFF rules. An organization's security policy can contain up to hundreds of rules. It is difficult to use all possible rules in experiment so a sample of rules is taken. After this the sampling procedure is identified. The important factors to consider in sampling are the sampling frame, method and the sample size. The sampling frame contains all members of the population that have the probability to be present in a sample and must be demonstrative of their population. In this thesis the sampling frame consisted of $14 \mathrm{VF}$ rules (strata $1 \& 2$ ). (Golnabi, 2006) formed a set of 7 unique firewall rules (strata 1) based on the generalization algorithm that showed all possible relations between all terms of the rule (Action, Protocol, Source Port, Destination Port, Source IP, Destination IP). The addition of 5 default rules and 2 rules to allow ping was done to the sample (strata 2). The final sample consisted of 14 rules. Due to the movement of Internet packets to and from VM at any given time, it is expected that at any given time the VM migration can be performed. Therefore, 8 migrations are performed in a row on $7^{\text {th }}$ January 2013 and used as the sample frame. The actual migration was scheduled in week before; due to technical issues the validation schedule was revised. 8 migrations, 4 migrations for each ping interval, were performed to wave off any validity threat of performing the experiment one time. For choosing a sampling method, it is essential to elect if probability sampling method is suitable or not. In it, all members of the population have the chance to be chosen in the sample. If members have the same probability to be selected, the sample can be unbiased. In this thesis it is possible to perform a random sampling with equal probabilities. Hence, the rules for a VM can be selected at random. Firewall rules between $2 \mathrm{VMs}$ in both to and from direction were selected which consist of IPs, ports and interfaces.

All records are selected using stratification i.e. complete strata 1 and 2. This means no rule selection was done from each stratum but rather entire strata were chosen. In this way, the sample can be said to have fairer representation of population. The sample rule-set is shown in Figure 4.

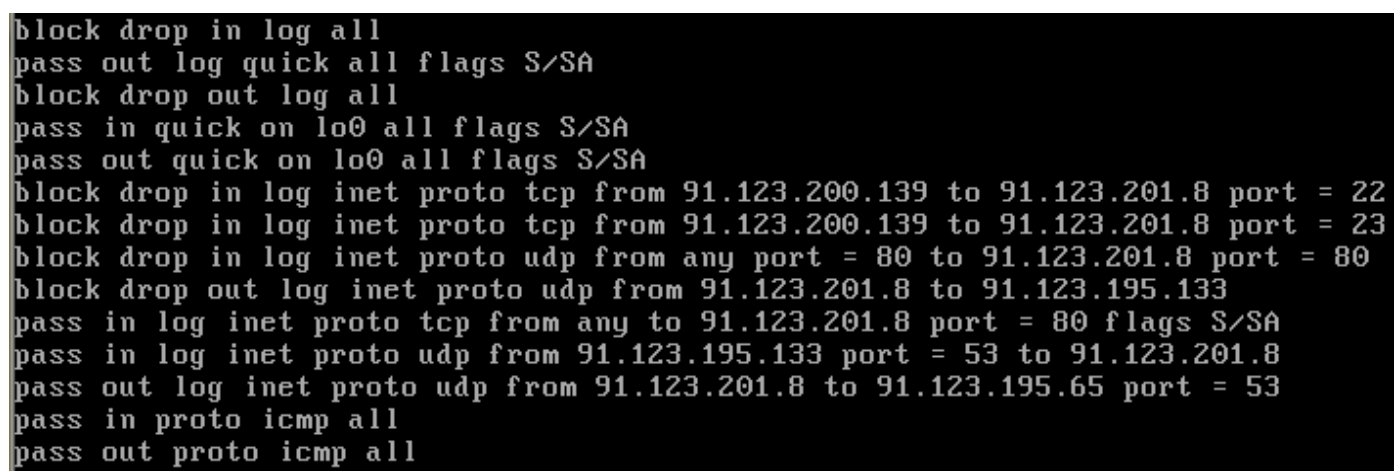

Figure 4: Sample PFF Rules

\subsection{Experiment Implementation}

To illustrate the potential of VM-level VF to protect the migrating VM, an implementation of open source PFF is done on VM. The implementation setup and 
choice of resources included the accessible resources at the City Network. The network in which the implementation is done consisted of physical hosts connected to internet through switch as in Figure 5. The hosts are connected to external storage and to other hosts using dedicated VLAN. Each host housed multiple VMs.

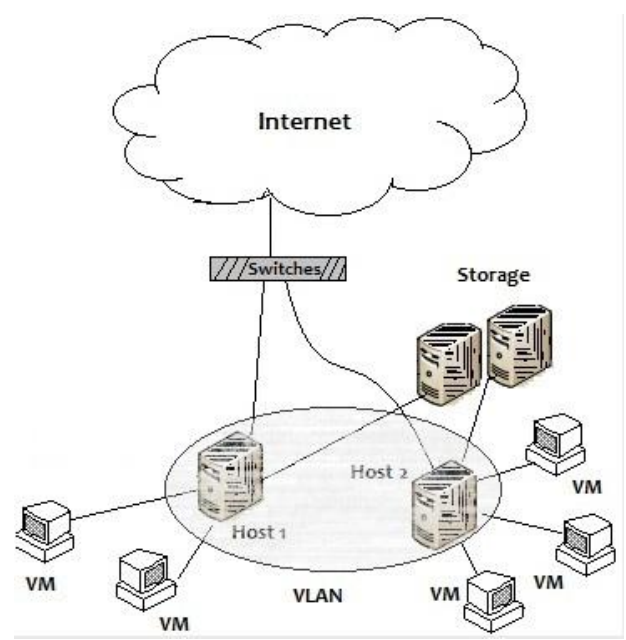

Figure 5: Cloud data center

The migration is executed on open source Kernel-based virtual machine monitor (KVM) residing on host computer using standard KVM migration algorithm. The algorithm is appended in Appendix A. QEMU ${ }^{12}$ (Quick emulator) is used to emulate hardware such as physical NIC for user. QEMU is accessed from Internet using Login name and password. Through successful Login user can access OS and data on VM. The block diagram for migration setup is shown in Figure 6. Figure 14 has migration interface.

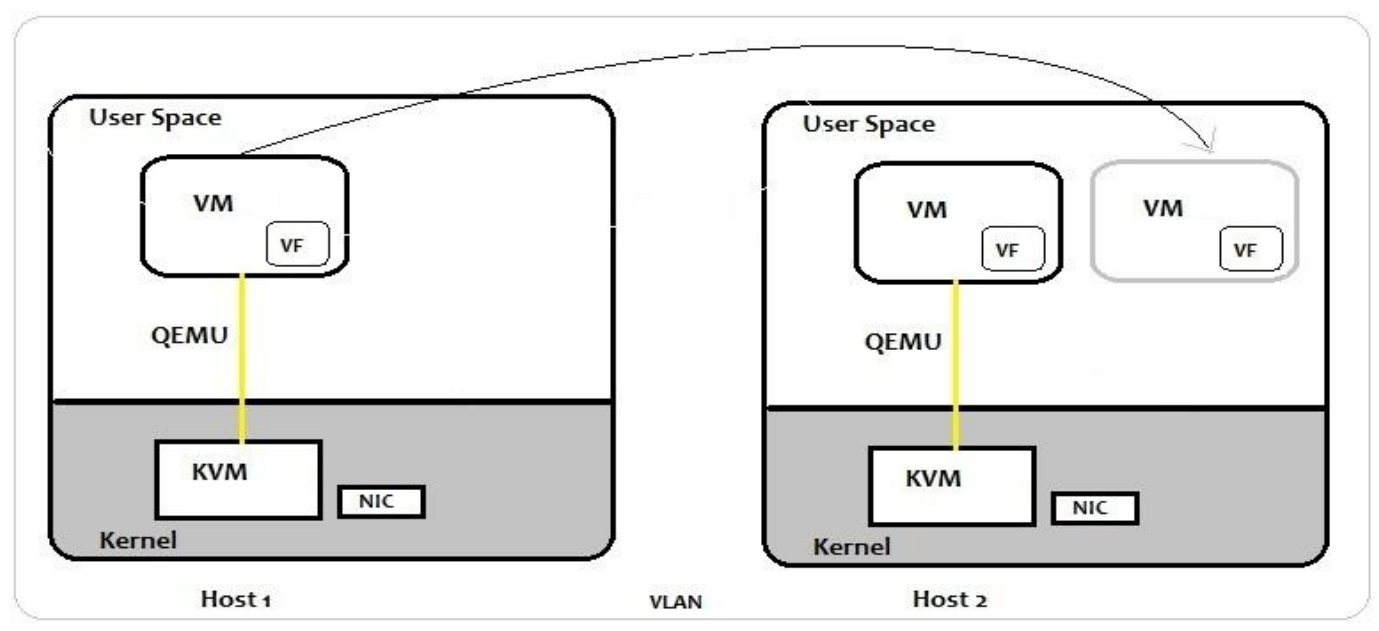

Figure 6: VM migration architecture

Appliances ranging from Linux flavors to Windows were available with hardware going from $1 \mathrm{CPU}$ to $8 \mathrm{CPUs}$ and varying memory choices from 10GB to $3000 \mathrm{~GB}$. To limit the scope of study VMs were prepared with similar specifications as in Table 5.

${ }^{12}$ http://wiki.qemu.org 
Table 5: Technical implementation specifications

\begin{tabular}{|l|l|}
\hline VM & \\
\hline Operating System & OpenBSD 5.1 64-bit \\
\hline CPU & 1 \\
\hline Memory & $512 \mathrm{MB}$ \\
\hline Network & $10 \mathrm{G} \mathrm{E}$ \\
\hline Host hardware & AMD64 \\
\hline CPU & 64GB and 128GB per node \\
\hline DRAM & Multiple 10G and multiple $1 \mathrm{G}$ \\
\hline NIC & \\
\hline VMM & KVM \\
\hline Name & OpenBSD 5.1 (Generic) \\
\hline Kernel Version & QEMU Virtual CPU 0.9.1 \\
\hline Emulator & \\
\hline
\end{tabular}

No migration optimization tools were used. The migration was performed using standard KVM migration. The implementation of set-up required:

1. Setting up 2 VMs (remote, target).

2. Configuring PFF policy on VMs.

a. Remote VM with policy that accepts all incoming and outgoing traffic.

b. Target VM with PFF sample rule-set that includes rules specified with IP, ports, interfaces and protocols; TCP, UDP, ICMP.

c. Rules are logged for analysis.

3. The VMM triggers the migration

The above steps are shown in Figure 11-13 in Appendix D.

\subsection{Experiment Validation Design}

After setting up VMs the VF was configured and loaded. Appendix E shows the communication between 2 VMs to verify the PFF rules function like they should. This was done before migration.

A session was setup with the migration expert. It was decided to perform live VM migrations to verify the valid functionality of the firewall rules during and after migration and to evaluate the intactness of VF using UT. The validation process was executed in Karlskrona, Sweden during 1500 hours from City Network. The day was chosen based on the availability of migration expert.

During each migration, the ping (ICMP) requests were sent and logged on remote system. The log file was also maintained on target VM to verify PFF behavior during migration. The reason to log packets on remote host has 2 reasons. One is to generate TCPDUMP on target VM to verify PFF functionality during migration. Second is to analyze the logs for missing ping packets for UT calculation. The log file on remote system captured target VM's IP, packet sequence number and time in milliseconds the packet took to get response from target VM.

For evaluation of PFF intactness the log on remote VM was analyzed to see total sent packets (for TMT) and missing packets (for DT). To validate PFF rule functionality after migration the rules were tested again on target VM. The results are shown in Appendix F. To have general results in total 8 migrations were done. The TMT and DT for migration were recorded for all VMs using the formulas. UT was calculated using formula. UT demonstrated the intactness of VF during migration, thus, showing the potential of VM-level VF during migration. The results are shown in Table 11. 
A pilot test was run to check if rules were functioning as desired. Test migration was performed as well to verify the PFF functionality before and after migration. After validation the data is recorded into Excel sheet. The following fields were recorded: VM ID, VM IP, VM OS, VM memory, VM CPU, host hardware, host CPU, VMM specification, VM rule set, VM rule-set functionality, ping packets send, ping packets received and ping sequence number. The system logs were stored in VMs (.txt and .cap format). It is useful to test each rule during migration but as migration slot is small enough to test all rules, only one was tested as shown in Figure 8 and Figure 9. If one rule was intact during migration it was assumed other rule for that specific run will also be intact.

\subsection{Experiment Verification}

To ensure that PFF rule-set functions as it should, the PFF was configured and tested between 2 VMs. The testing was done during preliminary test and also while doing validation. First, default rules were configured on PFF and their functionality verified. Then the individual rules of strata were tested separately. This was done because PFF matches the rules from top to bottom and fires the last matching rule. Doing separate verification for each rule ensured that rule was configured and loaded correctly. Then, all sample rules were loaded and functionality verified. Before the migration the IPs, VM IDs, and other data was recorded. All VMs were prepared for migration together. Each migration was done separately i.e. one VM was migrated at one time. Ping request was sent while VM transition to target VM from remote VM. Logs were maintained at remote VM. The process was repeated for all migrations to find out behavior of VF during VM motion. Lastly, the UT is calculated using TMT and DT difference and rules were verified again after VM migration.

Before commencing the validation process, migration expert at City Network tested the KVM migration. 


\section{VALIDITY THREATS}

The study faced several validity threats that threat the results and so must be catered before evaluating the use and validation of PFF in Cloud environment. Construct validity occurs when inadequate measures of variables are used (Creswell 2009). The design and reliability of experiment can have construct threat to the results. Results can be hampered with semantic error in firewall rule or incomplete packet transmission on network. To weaken the effect of the threat, rules published elsewhere were chosen for experimenting. Figure 4 shows how PFF configuration looked like. Further, ping interval was decreased to 0.01 seconds which means 100 packets were sent to target VM over network each second. It was done to send and capture many packets within a second to get more accurate number of missing packets which would result in precise and possibly near to actual uptime. This also potentially waved off the threat of using a simple network diagnostic tool, Ping. Making the interval smaller, more accuracy can be expected. Also to test the functionality of rule they were tested prior to running the actual migration experiment. The results of ping logs are shown in Table 11.

Other validity threats that can question the experiment's evaluation and validation are internal and external validity threats (Creswell 2009). Internal validity mirrors that the study's casual conclusion is justified. Such merit is founded by the extent to which the study reduces its bias. The external validity threat refers to wrong inference from the sample. The threat to these validities may occur if the PFF rules that are used in the evaluation are not representative of the population i.e. are biased in terms of size and rule functionality. If non-representative rules are chosen the result can be naive. To cater it, the stratified samples are used from population for the experiment. Both strata are included so all rule combinations can be included. Entire strata (strata $1+$ strata 2 ) were chosen for experimental validation to make it representative of the population. Furthermore, to reduce external validity threat on results due to any network latency or any other unknown factor the migrations were done 8 times along with statistical analysis; standard deviation and confidence interval. However, it is considered to replicate the study at later times with more number of migrations to determine if the same results occur.

The SLR was conducted without specifying start year to capture all the researches on the subject. However, using the key term, Cloud, could limit studies, as the term was used around 2008. This can be a threat of missing some studies before 2008. To reduce the threat the search was conducted on ACM without using key term 'Cloud'. 11 results were retrieved of which 8 were already the part of SLR. Other 3 were read and found unrelated to the topic. Similar result statistics were retrieved from Engineering Village and IEEE Xplore. Moreover, additional sources were reached to have broader SLR. Word 'security' was not used in SLR as key term as it resulted in many unrelated papers. Hence, the choice of 'firewall' was made without using any field preference. 


\section{RESULTS}

In this section, the answers to the research questions are presented.

\subsection{SLR Results}

\subsubsection{RQ1}

How much research is published on virtual firewalling for VM migration security in $C C$ ?

Table 6: Sources searched for all years (up to October 30 2012).

\begin{tabular}{|c|c|c|c|c|c|c|c|c|}
\hline Year & 2004 & 2007 & 2008 & 2009 & 2010 & 2011 & 2012 & Total \\
\hline \multicolumn{9}{|l|}{ ACM Digital } \\
\hline Relevant & 0 & 1 & 0 & 0 & 3 & 2 & 2 & 8 \\
\hline Selected & 0 & 0 & 0 & 0 & 2 & 1 & 1 & 4 \\
\hline \multicolumn{9}{|l|}{ CiteSeer } \\
\hline Relevant & 1 & 0 & 1 & 2 & 1 & 3 & 0 & 8 \\
\hline Selected & 0 & 0 & 1 & 0 & 1 & 0 & 0 & 2 \\
\hline \multicolumn{9}{|l|}{ IEEE Xplore } \\
\hline Relevant & 0 & 0 & 0 & 1 & 0 & 0 & 4 & 5 \\
\hline Selected & 0 & 0 & 0 & 0 & 0 & 0 & 3 & 3 \\
\hline \multicolumn{9}{|l|}{ Engineering Village } \\
\hline Relevant & 0 & 0 & 0 & 0 & 1 & 0 & 3 & 4 \\
\hline Selected & 0 & 0 & 0 & 0 & 1 & 0 & 2 & 3 \\
\hline \multicolumn{9}{|l|}{ Scopus } \\
\hline Relevant & 0 & 0 & 0 & 0 & 1 & 2 & 5 & 8 \\
\hline Selected & 0 & 0 & 0 & 0 & 1 & 1 & 3 & 5 \\
\hline \multicolumn{9}{|l|}{ ScienceDirect } \\
\hline Relevant & 0 & 1 & 0 & 0 & 0 & 0 & 0 & 1 \\
\hline Selected & 0 & 0 & 0 & 0 & 0 & 0 & 0 & 0 \\
\hline \multicolumn{9}{|l|}{ SpringerLink } \\
\hline Relevant & 0 & 0 & 0 & 0 & 0 & 0 & 0 & 0 \\
\hline Selected & 0 & 0 & 0 & 0 & 0 & 0 & 0 & 0 \\
\hline \multicolumn{9}{|l|}{ Web of Knowledge } \\
\hline Relevant & 0 & 0 & 0 & 0 & 0 & 1 & 1 & 2 \\
\hline Selected & 0 & 0 & 0 & 0 & 0 & 1 & 1 & 2 \\
\hline \multicolumn{9}{|l|}{$\begin{array}{l}\text { Wiley } \\
\text { Library }\end{array}$} \\
\hline Relevant & 0 & 0 & 0 & 0 & 0 & 0 & 0 & 0 \\
\hline Selected & 0 & 0 & 0 & 0 & 0 & 0 & 0 & 0 \\
\hline Total Relevant & 1 & 2 & 1 & 3 & 6 & 8 & 15 & 36 \\
\hline $\begin{array}{l}\text { Total Selected (with } \\
\text { duplicates) }\end{array}$ & 0 & 0 & 1 & 0 & 5 & 3 & 11 & 20 \\
\hline $\begin{array}{l}\text { Total Selected } \\
\text { (without duplicates) }\end{array}$ & 0 & 0 & 1 & 0 & 3 & 3 & 5 & 12 \\
\hline Other sources & 0 & 0 & 0 & 1 & 3 & 1 & 1 & 6 \\
\hline Final Selected & $\mathbf{0}$ & $\mathbf{0}$ & 1 & 1 & 6 & 4 & 6 & 18 \\
\hline
\end{tabular}

In Table 6 total relevant refers to papers that were retrieved from databases using search string. After applying inclusion/exclusion criteria on relevant studies the total selected studies were found. Papers from reference list of these selected studies were added to total selected and a set of final selected studies of SLR was formed. Duplicates mean when one paper is found in more than one database. Final selected studies are listed in Table 7 and

Table 8. Complete reference list is in Section 8.

Overall, 18 studies were identified that mentioned about securing VM when it migrates. Total 36 studies were identified from all databases as part of Stage 1 of study selection. Later, after de-duplication and applying the inclusion and exclusion criteria 12 studies were selected. On adding papers from reference list of selected studies the final selected studies rose to 18 . It can be seen that, most selected work on the topic occurred during or after 2008 with the most studies (67\%) published in 2010 and 2012.

2 studies (Jarraya, 2012; Jarraya, 2012(1)) referred to encoding and moving rules as algebraic terms for distributed firewalls. Thus, both studies directly relate to each 
other. (Tsugawa et al. 2010; Riteau 2011) implemented ViNe (virtual network) system to move VMs to different subnet without interruption in VM communication, and, maintaining firewall rules. 2 papers present how VMware secures VM migration (Zhou 2010; Basak et al. 2010). A system to detect unpatched firewall file on VMs in LAN was given by (Litty and Lie, 2011). (Xianqin et al. 2009) built a prototype system of the framework based on stateful firewall enabled Xen hypervisor. They suggest enhancing VMM capability to secure VM migration.

(Tavakoli et al. 2012) presented a framework for moving firewall policies in open source VMM. Their work was not evaluated. A framework for network virtualization to allow related VMs to be connected together on a virtual network, enforcing isolation security for group VM migrations was given by (Cabuk et al. 2008). Other secure migration frameworks was given by (Dawoud et al. 2010; Wu \& Winer 2010).

(Aslam et al. 2012) proposed Trusted Computing technique for secure VM launch and migration. The protocol guarantees that $\mathrm{VM}$ can only be migrated to a trustworthy cloud platform. The user defines the trust policy for VM like firewall rules. (Miaol et al., 2010) imitated network device called VSFilter which performed virtual firewalling to filter network packets. (Xianqin et al. 2009) emphasized on making VMM to tackle network attacks during VM mobility. (X. W. and Y. Chen 2011) discussed about securing communication mechanism during VM migration using software agents. However, (Szefer et al. 2011; Mann et al. 2012) presented the idea of reducing VMM work and suggested software-defined approach using OpenFlow protocol to secure migration. OpenFlow provided VF functionality. (Szefer et al. 2011) put the responsibility of VM-level security on customer.

Total 3 propositions were evaluated as in Table 8 . With respect to where the literature is published, ACM and Scopus included total 9 selected studies. Engineering Village and IEEE contributed to 3 studies each.

\subsubsection{RQ2}

What are the techniques addressed for virtual firewalling for VM migration security in CC?

Table 8 summarizes the techniques to provide firewall functionality to VM for secure migration. The detailed discussion on selected studies can be found in the papers. Table 8 presents each of the selected studies and categorizes them with firewall approach used for VM migration security, migration type, evaluation and limitations.

To summarize the selected studies, 7 papers talk about firewalls for migrating VM with VMM dependency. (Riteau 2011; Tsugawa et al. 2010) introduced virtual network to enhance the virtual network security and to ensure firewall connections move along with VM. For this to succeed, ViNe should be present on all VMMs. Another way to ensure tightened firewall rules is by automated detection and manual patching of vulnerable firewall configuration files (Litty \& Lie 2011). VMware way of ensuring virtual firewalling is by making use of their ESX VMM and vSheild firewall. The vMotion performs migration in coordination with vShiled firewall. The vShield manager ensures all firewalls are synchronized before migration (Zhou 2010; Basak et al. 2010). A similar virtual networking approach was given by (Cabuk et al. 2008; Dawoud et al. 2010). For open source VMM a framework was presented for migrating dynamic firewall rules with VM (Tavakoli et al. 2012). (Xianqin et al. 2009) built a prototype system of the framework based on stateful firewall enabled Xen hypervisor. They suggest enhancing VMM capability to secure VM migration. Of all these only 
(Basak et al. 2010) evaluated their work. (Szefer et al. 2011) was of the idea that VMlevel security is solely customer's responsibility.

Firewall rule preservation was discussed by selected SLR studies. (Jarraya, 2012; Jarraya, 2012(1)) referred to preservation of firewall rules after migration. They encoded the rule before migration and decoded after migration.

Some papers talked about other measures that mimicked firewall. (Aslam et al. 2012) proposed Trusted Computing technique for secure VM launch and migration. The protocol guarantees that VM can only be migrated to a trustworthy cloud platform. The user defines the trust policy for VM like firewall rules. (Miaol et al., 2010) imitated network device called VSFilter which performed virtual firewalling to filter network packets. (Xianqin et al. 2009) emphasized on making VMM to tackle network attacks during VM mobility. (X. W. and Y. Chen 2011) discussed about securing communication mechanism during VM migration using software agents. However, (Szefer et al. 2011; Mann et al. 2012) presented the idea of reducing VMM work and suggested software-defined approach using OpenFlow protocol to secure migration. OpenFlow provided VF functionality. (Szefer et al. 2011) put the responsibility of VM-level security on customer. 
Table 7: Selected studies

\begin{tabular}{|c|c|c|c|}
\hline ID & Reference and Year & Source & Title \\
\hline 1 & (Rexford 2012) & $\begin{array}{l}\text { Conference. } \\
\text { Proceedings of the } 39^{\text {th }} \text { Annual ACM SIGPLAN- } \\
\text { SIGACT Symposium on Principles of Programming } \\
\text { Languages (POPL) }\end{array}$ & $\begin{array}{l}\text { Programming Languages for Programmable } \\
\text { Networks }\end{array}$ \\
\hline 2 & (Szefer et al. 2011) & $\begin{array}{l}\text { Conference. } \\
\text { Proceedings of the 18th ACM conference on Computer } \\
\text { and communications security }\end{array}$ & $\begin{array}{l}\text { Eliminating the hypervisor attack surface for } \\
\text { a more secure Cloud }\end{array}$ \\
\hline 3 & (Litty \& Lie 2011) & $\begin{array}{l}\text { Conference. } \\
\text { Proceedings of the 7th ACM SIGPLAN/SIGOPS } \\
\text { international conference on Virtual execution } \\
\text { environments (VEE'11) }\end{array}$ & $\begin{array}{l}\text { Patch auditing in infrastructure as a service } \\
\text { Clouds }\end{array}$ \\
\hline 4 & (Zhou 2010) & $\begin{array}{l}\text { Journal } \\
\text { SIGOPS Operating Systems Review }\end{array}$ & Virtual networking \\
\hline 5 & (Basak et al. 2010) & $\begin{array}{l}\text { Journal. } \\
\text { Operating Systems Review (ACM) }\end{array}$ & $\begin{array}{l}\text { Virtualizing Networking and Security in the } \\
\text { Cloud }\end{array}$ \\
\hline 6 & (Riteau 2011) & $\begin{array}{l}\text { Conference. } \\
\text { International Symposium on Parallel and Distributed } \\
\text { Processing: Workshops and PhD Forum (IPDPS '11) }\end{array}$ & $\begin{array}{l}\text { Author manuscript, Building Dynamic } \\
\text { Computing Infrastructures over Distributed } \\
\text { Clouds }\end{array}$ \\
\hline 7 & (Tsugawa et al. 2010) & $\begin{array}{l}\text { Workshop. } \\
\text { GLOBECOM Workshops (GC Wkshps), } 2010 \text { IEEE }\end{array}$ & $\begin{array}{l}\text { User-level virtual networking mechanisms to } \\
\text { support virtual machine migration over } \\
\text { multiple Clouds }\end{array}$ \\
\hline 8 & $\begin{array}{l}\text { (Jarraya, A. Eghtesadi, } \\
\text { et al. 2012) }\end{array}$ & $\begin{array}{l}\text { Conference. } \\
\text { International Conference on Collaboration } \\
\text { Technologies and Systems (CTS) }\end{array}$ & $\begin{array}{l}\text { Cloud calculus: Security verification in } \\
\text { elastic Cloud computing platform }\end{array}$ \\
\hline 9 & $\begin{array}{l}\text { (Jarraya, Arash } \\
\text { Eghtesadi, et al. 2012) }\end{array}$ & $\begin{array}{l}\text { Conference. } \\
\text { Stabilization, Safety, and Security of Distributed } \\
\text { Systems. Proceedings of the 14th International } \\
\text { Symposium, SSS } 2012\end{array}$ & $\begin{array}{l}\text { Formal verification of security preservation } \\
\text { for migrating virtual machines in the Cloud }\end{array}$ \\
\hline 10 & (Tavakoli et al. 2012) & $\begin{array}{l}\text { Conference. } \\
\text { Information and } \\
\text { Proceedings 18th EUNICE/IFIP WG } \\
\text { International Conference, EUNICE 2012 }\end{array}$ & $\begin{array}{l}\text { A framework for security context migration } \\
\text { in a firewall secured virtual machine } \\
\text { environment. }\end{array}$ \\
\hline 11 & $\begin{array}{l}\text { (X. W. and Y. Chen } \\
\text { 2011) }\end{array}$ & $\begin{array}{l}\text { Journal. } \\
\text { Advances in Intelligent and Soft Computing }\end{array}$ & $\begin{array}{l}\text { Approach of the Secure Communication } \\
\text { Mechanism for the Off-Site Live Virtual } \\
\text { Machine Migrations }\end{array}$ \\
\hline 12 & (Cabuk et al. 2008) & $\begin{array}{l}\text { Conference. } \\
\text { The Rise and Rise of the Declarative Datacentre } \\
\text { May 12-13, 2008, Microsoft Research Cambridge, UK }\end{array}$ & $\begin{array}{l}\text { Declarative Security Specification of Virtual } \\
\text { Networks }\end{array}$ \\
\hline 13 & (Aslam et al. 2012) & $\begin{array}{l}\text { Conference. } \\
\text { IEEE International Conference on Trust, Security and } \\
\text { Privacy in Computing and Communications }\end{array}$ & $\begin{array}{l}\text { Security and Trust Preserving VM Migrations } \\
\text { in Public Clouds }\end{array}$ \\
\hline 14 & (Mann et al. 2012) & $\begin{array}{l}\text { Conference. } \\
\text { IEEE Network Operations and Management } \\
\text { Symposium }\end{array}$ & $\begin{array}{l}\text { CrossRoads: Seamless VM Mobility Across } \\
\text { Data Centers through Software Defined } \\
\text { Networking }\end{array}$ \\
\hline 15 & (Wu \& Winer 2010) & $\begin{array}{l}\text { Conference. } \\
\text { Seminar on Network Security, National Natural Science } \\
\text { Foundation of China }\end{array}$ & $\begin{array}{l}\text { Network Security for Virtual Machine } \\
\text { in Cloud Computing }\end{array}$ \\
\hline 16 & (Miaol et al. 2010) & $\begin{array}{l}\text { Conference. } \\
\text { International Conference on Educational and Network } \\
\text { Technology (ICENT) }\end{array}$ & $\begin{array}{l}\text { Developing a Virtual Network Environment } \\
\text { for Analyzing } \\
\text { Malicious Network Behavior }\end{array}$ \\
\hline 17 & (Dawoud et al. 2010) & $\begin{array}{l}\text { Conference. } \\
\text { The 7th International Conference on Informatics and } \\
\text { Systems }\end{array}$ & $\begin{array}{l}\text { Infrastructure as a Service Security: } \\
\text { Challenges and Solutions }\end{array}$ \\
\hline 18 & (Xianqin et al. 2009) & $\begin{array}{l}\text { Conference. } \\
\text { 2nd IEEE International Conference on Broadband } \\
\text { Network \& Multimedia Technology }\end{array}$ & $\begin{array}{l}\text { Seamless virtual machine live migration on } \\
\text { network security enhanced hypervisor }\end{array}$ \\
\hline
\end{tabular}


Table 8: Selected studies data.

\begin{tabular}{|c|c|c|c|c|c|c|}
\hline ID & $\begin{array}{c}\text { Firewall role in securing migrating } \\
\text { VM }\end{array}$ & Level & $\begin{array}{l}\text { Migra- } \\
\text { tion } \\
\text { Type: } \\
\text { LAN, } \\
\text { WAN }\end{array}$ & Limitations & $\begin{array}{l}\text { Evaluation for role of firewall } \\
\text { in securing VM migration }\end{array}$ & VMM \\
\hline 1,2 & $\begin{array}{l}\text { Security of Guest OS and applications } \\
\text { on it are customer's responsibility. } \\
\text { Migration model where customer } \\
\text { participates. } \\
\begin{array}{l}\text { Propose removing VMM so VM } \\
\text { directly accesses hardware. }\end{array}\end{array}$ & - & 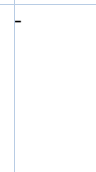 & Live VM migration as future work & - & None \\
\hline 3 & $\begin{array}{l}\text { System on VMM detects unpatched OS } \\
\text { and firewall. Upon detection system } \\
\text { reports to administrator. }\end{array}$ & VMM & - & $\begin{array}{l}\text { Overhead increases by } 4 \% \text {. } \\
\text { Delay in informing the VM } \\
\text { administrator. }\end{array}$ & - & Xen \\
\hline 4 & $\begin{array}{l}\text { VShield Firewall coordinates with } \\
\text { vMotion migration protocol to ensure } \\
\text { that the state travels with the VM. }\end{array}$ & VMM & LAN & $\begin{array}{l}\text { All hosts musts have vShield } \\
\text { Firewall. Firewall rules of VM are } \\
\text { sent to all possible hosts before } \\
\text { migration. }\end{array}$ & - & $\begin{array}{l}\text { VMWare } \\
\text { vSphere }\end{array}$ \\
\hline 5 & $\begin{array}{l}\text { Vmotion migrates VM. To move } \\
\text { firewall states all hosts must have } \\
\text { Vsheild. Vshield manager ensures } \\
\text { protection by sending VM rules to all } \\
\text { hosts. Vmotion should communicate } \\
\text { with Vmotion to have secure migration. }\end{array}$ & VMM & LAN & $\begin{array}{l}\text { Single choke point. } \\
\text { Extra load on hosts who get rules } \\
\text { of VM but VM never migrates to } \\
\text { that host. } \\
\text { No comparison with other VFs. }\end{array}$ & $\begin{array}{l}\text { Deploy } 10 \text { virtual security } \\
\text { firewall appliances } \text { (Edge } \\
\text { SVA) in parallel. Deploying } \\
\text { time: } \\
\text { Minimum: } 81 \mathrm{~s} \\
\text { Maximum:235s }\end{array}$ & $\begin{array}{l}\text { VMWare } \\
\text { vSphere } \\
\text { ESX }\end{array}$ \\
\hline 6,7 & $\begin{array}{l}\text { Implemented ViNe (virtual network) to } \\
\text { move VMs to } \\
\text { different subnet with interruption in } \\
\text { VM communication }\end{array}$ & VMM & LAN & $\begin{array}{l}\text { Dependency on VMM-to-VMM } \\
\text { communication. } \\
\text { Evaluation is future work. }\end{array}$ & - & KVM \\
\hline 8,9 & $\begin{array}{l}\text { The VM migration specifications and } \\
\text { firewall rules are encoded as algebra. } \\
\text { The encoding is compared for VM } \\
\text { before and after migration. Equivalence } \\
\text { depicts preservation of rules. }\end{array}$ & VMM & WAN & $\begin{array}{l}\text { No practical applicability and } \\
\text { performance overhead. }\end{array}$ & CPU time for VM migration & - \\
\hline 10 & $\begin{array}{l}\text { A framework is presented in which VM } \\
\text { firewall rules and other states are } \\
\text { moved with VM. }\end{array}$ & VMM & LAN & $\begin{array}{l}\text { Approach is not validated, nor } \\
\text { evaluated. }\end{array}$ & - & KVM \\
\hline 11 & Software agents perform migration. & - & WAN & $\begin{array}{l}\text { Communication between agents } \\
\text { can be vulnerable. }\end{array}$ & - & - \\
\hline 12 & $\begin{array}{l}\text { Presented framework for network } \\
\text { virtualization to allow related VMs to } \\
\text { be connected together on a virtual } \\
\text { network, enforcing isolation security } \\
\text { for group VMs during migration. }\end{array}$ & VMM & LAN & $\begin{array}{l}\text { VMs in same group can securely } \\
\text { migrate }\end{array}$ & - & - \\
\hline 13 & $\begin{array}{l}\text { Proposed alternate with Trusted } \\
\text { Computing technique for secure VM } \\
\text { launch and migration. The protocol } \\
\text { guarantees that VM can only be } \\
\text { migrated to a trustworthy cloud } \\
\text { platform. }\end{array}$ & VMM & - & $\begin{array}{l}\text { Applicable with } \quad \text { Trusted } \\
\text { Computing Module only. }\end{array}$ & - & - \\
\hline 14 & $\begin{array}{l}\text { Leverage software defined networking } \\
\text { and implement an OpenFlow based } \\
\text { prototype for seamless live VM } \\
\text { mobility across multiple data centers }\end{array}$ & $\begin{array}{l}\text { Central } \\
\text { control } \\
\text { plane }\end{array}$ & WAN & - & Evaluated performance & - \\
\hline 15 & $\begin{array}{l}\text { Presented Virtual network framework } \\
\text { to improve security of the inter- } \\
\text { communication among VMs. Used } \\
\text { firewall to protect from sniffing and } \\
\text { spoofing attacks on VM. }\end{array}$ & VMM & LAN & - & - & Xen \\
\hline 16 & $\begin{array}{l}\text { Filters packets and forward allowed } \\
\text { packets to destination VMM }\end{array}$ & - & LAN & - & - & - \\
\hline 17 & $\begin{array}{l}\text { Has architecture for secure } \\
\text { provisioning and migration of } \\
\text { VMs. They focus on Private Virtual } \\
\text { Infrastructure for monitoring, tamper } \\
\text { detection and secure shutdown for } \\
\text { migration. }\end{array}$ & VMM & LAN & - & - & - \\
\hline 18 & $\begin{array}{l}\text { Emphasize on network security enabled } \\
\text { VMM to protect VM from virtual } \\
\text { network attacks using stateful firewall. }\end{array}$ & VMM & LAN & - & $\begin{array}{l}\text { Download file using } \text { FTP. } \\
\text { The file downloads with } \\
\text { DT=1.6s. }\end{array}$ & Xen \\
\hline
\end{tabular}

Here a hyphen denotes that no data is available. 


\subsubsection{RQ3}

Are the researches limited?

To answer it 2 sub-questions were considered which are as follows:

RQ3.1. Are the research topics limited?

RQ3.2. What is the quality of literature?

Table 9 gives list of 13 papers not selected in the SLR i.e. 52\% of relevant literature did not focus on the subject. Instead they covered other aspects of VM migration such as enhancing resource sharing with migration, optimizing VM migration performance or virtualization security in general. None of them focused on securing VM during migration or testing firewall behavior during VM migration in CC.

Table 9: Candidate studies not selected

\begin{tabular}{|c|c|c|c|c|}
\hline ID & Source & Reference and Year & Title & $\begin{array}{l}\text { Exclusion } \\
\text { reason }\end{array}$ \\
\hline 1 & $\begin{array}{l}\text { In Proceedings of the 7th ACM } \\
\text { SIGPLAN/SIGOPS international conference } \\
\text { on Virtual execution environments (VEE '11) }\end{array}$ & (Wood \& Shenoy 2011) & $\begin{array}{l}\text { CloudNet: dynamic pooling of } \\
\text { Cloud resources by live WAN } \\
\text { migration of virtual machines }\end{array}$ & $\begin{array}{l}\text { Exclusion } \\
\text { criteria } 3\end{array}$ \\
\hline 2 & $\begin{array}{l}\text { In Proceedings of the 9th USENIX } \\
\text { conference on Operating systems design and } \\
\text { implementation (OSDI'10) }\end{array}$ & $\begin{array}{l}\text { (Timothy Broomhead, } \\
\text { Laurence } \quad \text { Cremean, } \\
\text { Julien Ridoux 2010) }\end{array}$ & Virtualize everything but time & $\begin{array}{l}\text { Exclusion } \\
\text { criteria } 3\end{array}$ \\
\hline 3 & $\begin{array}{l}\text { In Proceedings of the 4th USENIX } \\
\text { conference on Networked systems design and } \\
\text { implementation (NSDI'07) }\end{array}$ & (Wood et al. 2007) & $\begin{array}{l}\text { Black-box and gray-box strategies } \\
\text { for virtual machine migration }\end{array}$ & $\begin{array}{l}\text { Exclusion } \\
\text { criteria } 3\end{array}$ \\
\hline 4 & $\begin{array}{l}\text { Proceedings of the 23rd conference on Large } \\
\text { installation system administration (LISA'09) }\end{array}$ & (Tripathi et al. 2009) & $\begin{array}{l}\text { Crossbow virtual wire: network in a } \\
\text { box }\end{array}$ & $\begin{array}{l}\text { Exclusion } \\
\text { criteria } 3\end{array}$ \\
\hline 5 & $\begin{array}{l}\text { Proceedings of the 1st ACM workshop on } \\
\text { Virtualized infrastructure systems and } \\
\text { architectures (VISA } 09 \text { ) }\end{array}$ & (Hao et al. 2009) & $\begin{array}{l}\text { Enhancing Dynamic Cloud-based } \\
\text { Services } \\
\text { using Network Virtualization }\end{array}$ & $\begin{array}{l}\text { Exclusion } \\
\text { criteria } 3\end{array}$ \\
\hline 6 & $\begin{array}{l}\text { Proceedings of the 7th ACM } \\
\text { SIGPLAN/SIGOPS international conference } \\
\text { on Virtual execution environments }\end{array}$ & (Wood \& Shenoy 2010) & $\begin{array}{l}\text { CloudNet: A Platform for Optimized } \\
\text { WAN Migration of Virtual } \\
\text { Machines }\end{array}$ & $\begin{array}{l}\text { Exclusion } \\
\text { criteria } 3\end{array}$ \\
\hline 7 & $\begin{array}{l}\text { Proceedings of the } 2011 \text { International } \\
\text { Conference on Parallel Processing (ICPP '11) }\end{array}$ & (Xu et al. 2011) & $\begin{array}{l}\text { WAVNet: Wide-Area Network } \\
\text { Virtualization Technique for Virtual } \\
\text { Private Cloud }\end{array}$ & $\begin{array}{l}\text { Exclusion } \\
\text { criteria } 3\end{array}$ \\
\hline 8 & $\begin{array}{l}\text { Journal Computer Virology, Volume } 8 \text {, No. } 3 \\
\text { (August 2012) }\end{array}$ & (Ranjith et al. 2012) & $\begin{array}{l}\text { On covert channels between virtual } \\
\text { machines }\end{array}$ & $\begin{array}{l}\text { Exclusion } \\
\text { criteria } 3\end{array}$ \\
\hline 9 & $\begin{array}{l}\text { Proceedings of the 16th European conference } \\
\text { on Research in computer security } \\
\text { (ESORICS'11) }\end{array}$ & $\begin{array}{l}\text { (Schunter \& Eriksson } \\
\text { 2011) }\end{array}$ & $\begin{array}{l}\text { Automated information flow } \\
\text { analysis of virtualized infrastructures }\end{array}$ & $\begin{array}{l}\text { Exclusion } \\
\text { criteria } 3\end{array}$ \\
\hline 10 & $\begin{array}{l}\text { Proceedings of the 6th international workshop } \\
\text { on Virtualization Technologies in Distributed } \\
\text { Computing Date (VTDC '12) }\end{array}$ & (Stabler et al. 2012) & $\begin{array}{l}\text { Elastic IP and security groups } \\
\text { implementation using OpenFlow }\end{array}$ & $\begin{array}{l}\text { Exclusion } \\
\text { criteria } 3\end{array}$ \\
\hline 11 & $\begin{array}{l}\text { Component Deployment: Second } \\
\text { International Working Conference (LNCS } \\
\text { volume 3083/2004) }\end{array}$ & (Cutsem et al. 2004) & $\begin{array}{l}\text { On the Performance of SOAP in a } \\
\text { Non-Trivial } \\
\text { Peer-to-Peer Experiment }\end{array}$ & $\begin{array}{l}\text { Exclusion } \\
\text { criteria } 3\end{array}$ \\
\hline 12 & $\begin{array}{l}\text { IEEE International Symposium on Network } \\
\text { Computing and Applications }\end{array}$ & (Miyamoto et al. 2009) & $\begin{array}{l}\text { Customizing Network Functions for } \\
\text { High Performance Cloud Computing }\end{array}$ & $\begin{array}{l}\text { Exclusion } \\
\text { criteria } 3\end{array}$ \\
\hline 13 & IEEE Communications Magazine & (Decusatis et al. 2012) & $\begin{array}{l}\text { Communication within Clouds: } \\
\text { Open Standards and Proprietary } \\
\text { Protocols for Data Center } \\
\text { Networking }\end{array}$ & $\begin{array}{l}\text { Exclusion } \\
\text { criteria } 3\end{array}$ \\
\hline
\end{tabular}

Out of the selected studies a trend was seen of having VMM-level firewall security to protect VMs while migration. Moreover, the papers that covered some sort of virtual firewalling were either not evaluated or dealt with commercial solutions. Thirdly, these results did not evaluate the intactness or behavior of VF during migration.

Security policies are deployed in form of firewall rules in Cloud data center to enable security. This prevents VM from migration related attacks as discussed above. The physical and VMM-level firewalls cannot protect VM in virtual network or during migration. Plus, VMM-level VF may not always be customized for specific VM and can also be latent to secure migrating VM. When VM migrates, it is vulnerable to attacks such as VM fake migration, session hijacking, loss of service and etc. It thus, 
becomes critical to ensure firewall policy intactness on VM during migration and evaluate it during migration.

To answer RQ3.2 the quality assessment method of (Barbara 2007) is used. The quality questions are defined in Table 4 . The score for each study is shown in Table 10. Study quality varied from 2 to 5 , where a quality value of 5 is highest, as measured from the 5 questions in Table 4 . The average study quality is $3.6(68 \%)$, which shows that most of the selected studies were of high quality. None exhibited intactness of VF during migration and no open source VFs at VM-level was researched. They tended to lack the empirical evaluation on subject and some did not discuss the limitations of approach.

Table 10: Study Quality

\begin{tabular}{|l|l|l|l|l|l|l|}
\hline ID & QA1 & QA2 & QA3 & QA4 & QA5 & Total score \\
\hline 1 & 0.5 & 0 & 1 & 0 & 0.5 & 2 \\
\hline 2 & 0.5 & 1 & 1 & 0 & 1 & 4.5 \\
\hline 3 & 1 & 1 & 1 & 0 & 1 & 5 \\
\hline 4 & 0.5 & 0 & 1 & 0 & 1 & 2.5 \\
\hline 5 & 1 & 1 & 1 & 1 & 1 & 5 \\
\hline 6 & 0.5 & 0 & 1 & 0 & 0.5 & 2 \\
\hline 7 & 1 & 1 & 1 & 0 & 1 & 4 \\
\hline 8 & 0.5 & 1 & 0.5 & 1 & 1 & 3 \\
\hline 9 & 0.5 & 1 & 0.5 & 1 & 1 & 3 \\
\hline 10 & 1 & 1 & 0.5 & 0 & 1 & 3.5 \\
\hline 11 & 0.5 & 0.5 & 1 & 0 & 1 & 3 \\
\hline 12 & 0.5 & 1 & 1 & 1 & 1 & 4.5 \\
\hline 13 & 1 & 1 & 1 & 1 & 1 & 5 \\
\hline 14 & 0.5 & 1 & 0 & 1 & 1 & 3.5 \\
\hline 15 & 1 & 1 & 0 & 1 & 1 & 4 \\
\hline 16 & 1 & 1 & 0 & 1 & 1 & 4 \\
\hline 17 & 1 & 1 & 0 & 1 & 1 & 4 \\
\hline 18 & 1 & 1 & 0 & 1 & 1 & 4 \\
\hline
\end{tabular}

\subsection{Experiment Validation and Evaluation Results}

\subsubsection{RQ4}

Does VF at VM-level protect the VM during migration and what is its empirical evaluation?

The results of the 8 live migrations are gathered with the help of migration expert at City Network. To view the firewall rule intactness during migration ICMP (ping) packets were sent from a remote system to target VM while it was in transition. The ping requests were logged in file on the remote machine to capture number of ICMP packets that were missed. The firewall rule is configured on target machine to pass in and out the ping requests. Table 11 depicts the total number of ICMP requests sent and replies received for each VM which was used to measure downtime and approximate TMT of VM. VM downtime is assumed to be equal to VF downtime. On subtracting VF DT from TMT, the active/up time for VF can be measured.

Table 11: Firewall intactness evaluation using ICMP packets

For ICMP packet transmission interval $=0.5 \mathrm{~s} \backslash$

\begin{tabular}{|c|c|c|c|c|c|c|c|c|c|}
\hline$\overline{\text { VM ID }}$ & $\begin{array}{l}\text { Uptime } \\
\text { (UT = } \\
\text { TMT-DT) }\end{array}$ & $\begin{array}{l}\text { Downtime } \\
(\text { DT }=\text { I*PL })\end{array}$ & \multicolumn{2}{|c|}{$\begin{array}{l}\text { Packets lost } \\
(\mathbf{P L}=\text { PS-PR })\end{array}$} & $\begin{array}{l}\text { Packets } \\
\text { Sent } \\
\text { (PS) }\end{array}$ & \multicolumn{2}{|c|}{$\begin{array}{l}\text { Packets } \\
\text { Received (PR) }\end{array}$} & $\begin{array}{l}\text { Total Migration } \\
\text { Time } \\
\text { (TMT=PS/2 s) }\end{array}$ & VM IP \\
\hline VM1 & 83 & 1 & 2 & & 168 & 166 & & 84 & 91.123 .201 .8 \\
\hline VM2 & 45.5 & 0.5 & 1 & & 92 & 91 & & 46 & 5.184 .38 \\
\hline VM3 & 51 & 0.5 & 1 & & 103 & 102 & & 51.5 & 5.189 .185 \\
\hline VM4 & 29.5 & 1 & 2 & & 61 & 59 & & 30.5 & 141.255 .188 .144 \\
\hline \multicolumn{2}{|c|}{ Mean DT $=0.75 \mathrm{~s}$} & \multicolumn{2}{|c|}{ DT Variance $=0.08 \mathrm{~s}$} & \multicolumn{3}{|c|}{$\begin{array}{l}\text { DT Standard Deviation } \\
=0.28 \mathrm{~s}\end{array}$} & \multicolumn{3}{|c|}{$\begin{array}{l}\text { DT Confidence Interval } \\
=0.5 \mathrm{~s} \text { to } 1.0 \mathrm{~s}(95 \% \text { confidence })\end{array}$} \\
\hline \multicolumn{2}{|c|}{ Mean TMT $=53 \mathrm{~s}$} & \multicolumn{2}{|c|}{$\begin{array}{l}\text { TMT Variance }=506.16 \\
\mathrm{~s}\end{array}$} & \multicolumn{3}{|c|}{$\begin{array}{l}\text { TMT Standard Deviation } \\
=22.49 \mathrm{~s}\end{array}$} & \multicolumn{3}{|c|}{$\begin{array}{l}\text { TMT Confidence Interval } \\
=30.5 \mathrm{~s} \text { to } 75.5 \mathrm{~s}(95 \% \text { confidence })\end{array}$} \\
\hline \multicolumn{2}{|c|}{ Mean UT $=52.25 \mathrm{~s}$} & \multicolumn{2}{|c|}{ UT Variance $=503.4 \mathrm{~s}$} & \multicolumn{3}{|c|}{$\begin{array}{l}\text { UT Standard Deviation } \\
=22.43 \mathrm{~s}\end{array}$} & \multicolumn{3}{|c|}{$\begin{array}{l}\text { UT Confidence Interval } \\
=30.3 \mathrm{~s} \text { to } 74.15 \mathrm{~s}(95 \% \text { confidence })\end{array}$} \\
\hline
\end{tabular}


For ICMP packet transmission interval $=0.01 \mathrm{~s}$

\begin{tabular}{|c|c|c|c|c|c|c|c|c|c|}
\hline VM ID & $\begin{array}{l}\text { Uptime } \\
\text { (UT = } \\
\text { TMT - DT) }\end{array}$ & $\begin{array}{l}\text { Downtime } \\
(\mathrm{DT}=\mathbf{I} * \mathbf{P L})\end{array}$ & \multicolumn{2}{|c|}{$\begin{array}{l}\text { Packets lost } \\
(\text { PL = PS-PR) }\end{array}$} & $\begin{array}{l}\text { Packets } \\
\text { Sent } \\
\text { (PS) }\end{array}$ & \multicolumn{2}{|c|}{$\begin{array}{l}\text { Packets } \\
\text { Received (PR) }\end{array}$} & $\begin{array}{l}\text { Total Migration } \\
\text { Time } \\
\text { (TMT=PS/100 s) }\end{array}$ & VM IP \\
\hline VM5 & 11.8 & 0.52 & \multicolumn{2}{|l|}{52} & 1240 & \multicolumn{2}{|l|}{1188} & 12.4 & 91.123 .200 .33 \\
\hline VM6 & 14.67 & 0.53 & \multicolumn{2}{|l|}{53} & 1527 & \multicolumn{2}{|l|}{1474} & 15.2 & 91.123 .201 .50 \\
\hline VM7 & 8.45 & 0.55 & \multicolumn{2}{|l|}{55} & 903 & \multicolumn{2}{|l|}{848} & 9.0 & 188.95 .224 .175 \\
\hline VM8 & 30.1 & 0.3 & \multicolumn{2}{|l|}{30} & 3070 & \multicolumn{2}{|l|}{3040} & 30.4 & 91.123 .200 .139 \\
\hline \multicolumn{2}{|c|}{ Mean DT $=0.47 \mathrm{~s}$} & \multicolumn{2}{|c|}{ DT Variance $=0.01 \mathrm{~s}$} & \multicolumn{3}{|c|}{ DT Standard Deviation $=0.11 \mathrm{~s}$} & \multicolumn{3}{|c|}{$\begin{array}{l}\text { DT Confidence Interval } \\
=0.37 \mathrm{~s} \text { to } 0.57 \mathrm{~s}(95 \% \text { confidence })\end{array}$} \\
\hline \multicolumn{2}{|c|}{ Mean TMT $=16.6 \mathrm{~s}$} & \multicolumn{2}{|c|}{ TMT Variance $=89.24 \mathrm{~s}$} & \multicolumn{3}{|c|}{ TMT Standard Deviation $=9.44 \mathrm{~s}$} & \multicolumn{3}{|c|}{$\begin{array}{l}\text { TMT Confidence Interval } \\
=9.3 \mathrm{~s} \text { to } 25.9 \mathrm{~s}(95 \% \text { confidence })\end{array}$} \\
\hline \multicolumn{2}{|c|}{ Mean UT $=16.25 \mathrm{~s}$} & \multicolumn{2}{|c|}{ UT Variance $=68.73 \mathrm{~s}$} & \multicolumn{3}{|c|}{ UT Standard Deviation $=8.29 \mathrm{~s}$} & \multicolumn{3}{|c|}{$\begin{array}{l}\text { UT Confidence Interval } \\
=8.13 \mathrm{~s} \text { to } 24.37 \mathrm{~s}(95 \% \text { confidence })\end{array}$} \\
\hline
\end{tabular}

Here Uptime (for $\mathrm{I}=0.01 \mathrm{~s}$ ) varies from $93 \%$ to $99 \%$. In other words it means that for up to $99 \%$ of migration span the VF at VM-level was found to be blocking and allowing packets i.e. it was intact.

When the ping interval was set to half of a second, the ICMP requests were sent 2 times in each second. As the number of packets loss is always a whole number, the downtime value will always be a whole number or nearest to 0.5 . To get more precise results the ping interval was decreased to a $100^{\text {th }}$ part of a second i.e. 100 ping requests were sent per second. This allowed to send and to capture more packets per second which resulted in more tuned value of packets lost per second as in Table 11. Having decreased the interval gave mean downtime equal to $0.47 \mathrm{~s}$, as compared to $0.75 \mathrm{~s}$ with large interval.

The table shows mean, standard deviation and confidence interval for DT, TMT and UT. The standard deviation enables to depict which VMs have their UT within a standard deviation of mean. It shows that $87 \%$ of the values are within a standard deviation of a mean and can be classified as normal i.e. most of the VMs have normal uptime.

With 5\% risk of being wrong (95\% confidence) the UT of machines varied between $8 \mathrm{~s}$ and $24 \mathrm{~s}$, with mean UT $16.25 \mathrm{~s}$ (for Ping Interval $0.01 \mathrm{~s}$ ). It can be said that if VF is implemented on VM, 97\% of the migration interval has active firewall protection (here mean TMT is $16.6 \mathrm{~s}$ ). Having 97\% protection of migration slot, reduces attacker's chances to gain access to VM. This is presented in Figure 7.

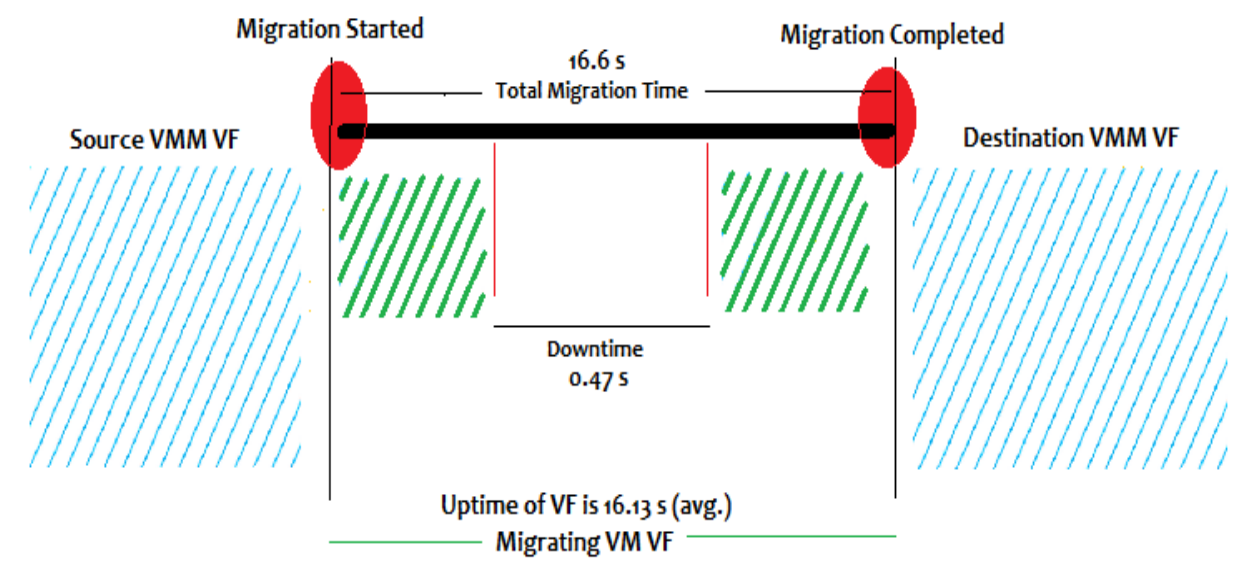

Figure 7: VF Uptime 
To test if of PF rules were intact during transition, TCPDUMP logs were captured on target VM. However, the PPF rules were also tested after migration to verify the functionality of the rule and were found to be correctly configured.

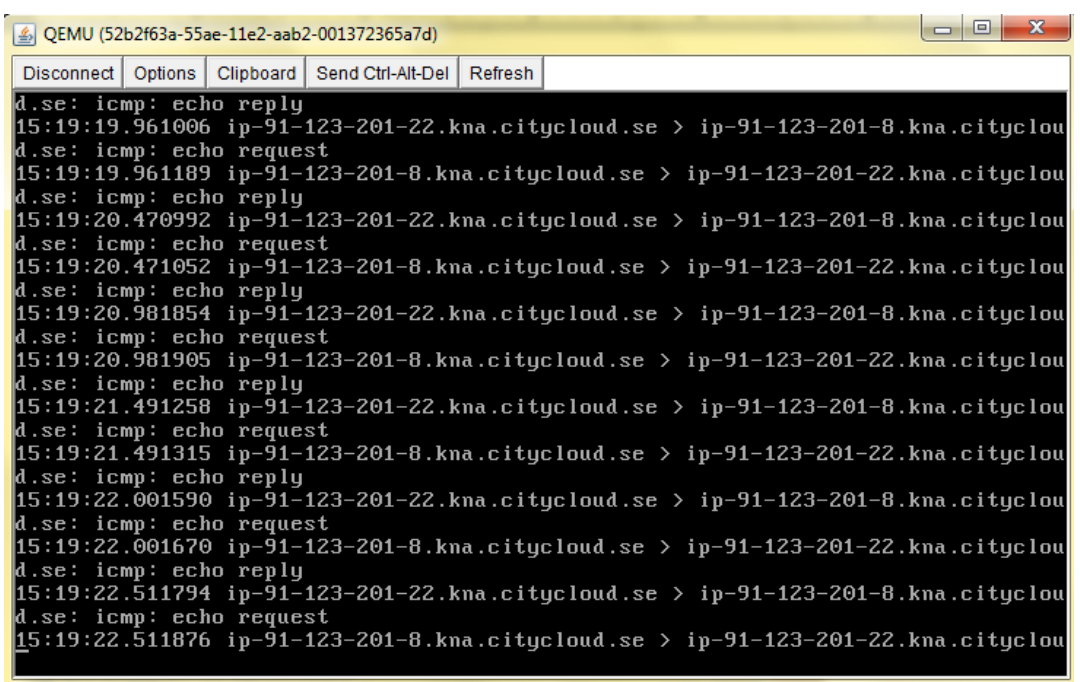

Figure 8: Target VM TCPDUMP log.

On 19th minute 20th second and 22nd second, 4 ICMP requests and responses were logged. The ping interval is 0.5 seconds, in this case. On 19th minute at $21 \mathrm{st}$ second target VM (91.123.201.8) received a packet request. 2 packets are missing in the logs.

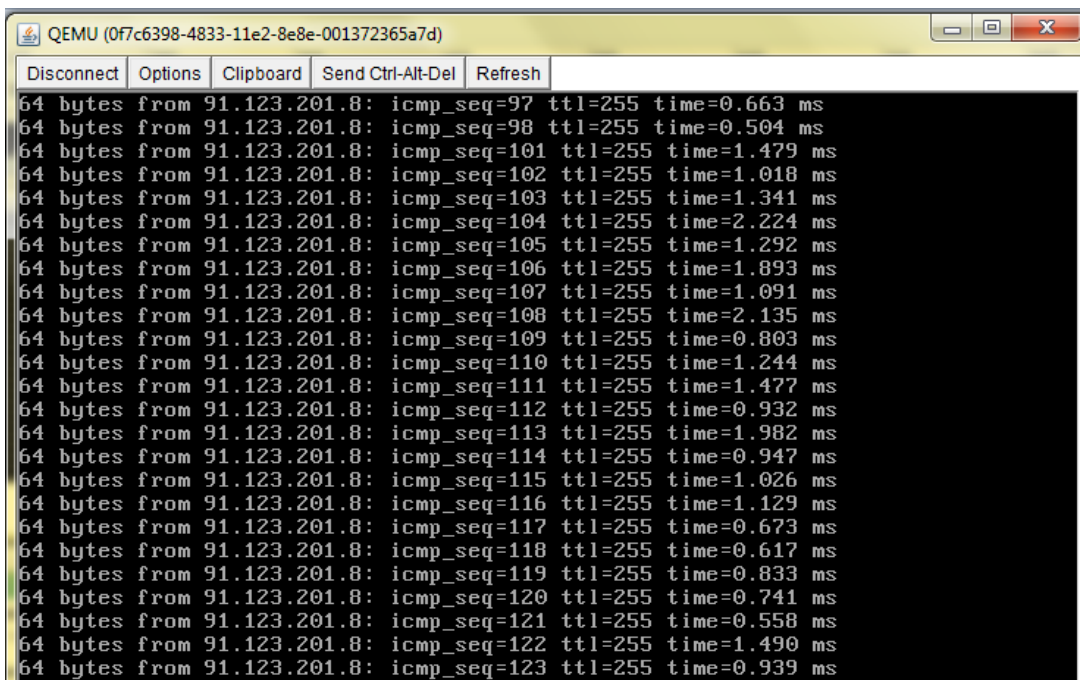

Figure 9: Remote VM ping log

VM (91.123.201.22) sends ICMP requests to target VM (IP: 91.123.201.8). ICMP sequence shows 2 missing packets (icmp_seq 99 and 100). 


\section{DISCUSSION}

Making use of VF at VM-level will help the VM customers to enhance the VM security during migration. As the SLR showed there are studies that mention about VF at VMM-level using virtual network system (Tsugawa et al. 2010) or a virtual firewall VM as in (Basak et al. 2010) or enhancing the security of VMM as in (Xianqin et al. 2009). In the majority of studies idea to secure VM relied on VMM-level security or having commercial products. None measured VF activeness during migration. They lacked investigation on using open source virtual firewalling solution at VM-level. Therefore, in this study VF is implemented at VM-level with help of data center experts to research on the prospective of VF such as OpenBSD PFF at VM-level as a complement to the other available firewalls in virtual set-up. Also uptime is calculated to evaluate its activeness, thus, filling up research gap.

The behavior of VF is evaluated and validated using an experiment which depicted mean uptime of $16.2 \mathrm{~s}$ (for I=0.01s). The migration expert evaluated the validity of the results in real data center. Knowing the fact that no optimization was used to perform virtual firewalling or for migration, the downtime was found close to VMware's product ( $0.4 \mathrm{~s}$ approximately) (Salfner et al. 2011) which shows the validity of open source virtual firewalling at VM-level. In (Salfner et al. 2011) VMware analysis of downtime is done for $4 \mathrm{~GB}$ machine with working set equal to 0MB. As target machine was idle, we assume the working set was close to OMB. It is assumed, as VF DT is close to VMware's, the UT calculation would also be close to other products in industry.

Additionally, no studies reported empirical evaluation of VF such as PFF during $\mathrm{VM}$ migration at VM-level in CC which refers to the research gap in the research industry. However, the diagnostic network tool Ping used for calculation of uptime may not be a best choice for evaluating critical live migration process. It is considered to run similar experiment using other available network statistic tool.

Although the PFF rules used as experiment sample are taken from a research paper (Golnabi et al. 2006), using more terms in rule may alter the results. While performing rule configuration, it is however, critical for administrator to have firewalling knowledge. PFF, although provide tightened security, but in case of false or incomplete rule the firewall can produce incorrect behavior. Often misconfiguration leads to administrators' lack of motivation. To cater this, the idea is to marry use of VMM-level firewalling, to add up a security layer providing VMs from disastrous attacks. However, for expert user, this may not hold true. With this, PF provides logging feature at a very grain level so getting additional data can be useful, for security professionals, which can be utilized to detect intrusion attempts and attack patterns.

Having VM-level VF resolves other issues such as dependency on Cloud provider to maintain fine grained VM security. As VMM is more prone to attacks than VM, hence, the security mechanism on VM naturally keeps VM safer than having security only on VMM.

In small companies and businesses where budget is not too high for security, using this approach for VM security during migrations can leverage the owner from licensing cost and up-gradation costs of using commercial services. Moreover, when it comes to secure OS, OpenBSD tops up. Even, in terms of firewalling features and security perspective OpenBSD PFF holds good repute. 
Third party can provide firewall-as-a-service for $\mathrm{CC}$ users and manage firewall for customers who do not have the resources to manage it themselves for example complex security policies. 


\section{CONCLUSION AND FUTURE WORK}

This research looked into the potential of virtual firewalling at VM-level using open source Packet filter firewall as an additional security layer for migrating virtual machine's (VM). A systematic literary review was done according to which the VMlevel security is not researched enough, nor is evaluated during migration. Based on related work, the design was set-up and firewalling done for VM. To test its functionality and uptime an experiment with the help of migration expert was executed. The firewall was found to be intact during migration process with mean uptime of $16.25 \mathrm{~s}(\mathrm{I}=0.01 \mathrm{~s})$. The reason for change in uptime is not a part of this research.

The validity of the results was examined with the help of the data center migration expert from City Network, Sweden. The validation process showed that the $75 \%$ of the uptime values were normal uptime ranges (in this scenario). Additionally, a potential research area was found during the validation process. Although, the VMs were not under heavy workload, even then, some had more round trip ping time. It can be looked in future, the reasons which caused the round trip time to vary greatly. Also, it was found that the maximum round time occurred before packet loss in some cases. The study highlighted the understanding of using VM-level open source VF for VM migration security and VM security in general as a supplement to the VMM-level firewalling in CC. It is concluded that using such an approach will likely bring improved security for VMs like an additional security perimeter layer on the network and providing customized and deep security.

It is however, essential to perform a similar experiment using more sophisticated network diagnostic tools. To overcome the shortcomings of having 8 migrations, another similar study with more migrations can further validate its results. It's also beneficial to use PFF with full security features and test in real environment for days to verify its resiliency. In the research, the migration was done in LAN. It can open doors to a common gate for doing secure WAN migrations; hence, looking into WAN migrations in future can be a yielding prospect. There are other virtual security appliances that can leverage VM security and security of virtualization architecture on the whole. The rules used to perform experiment were simple i.e. either had port blocking or protocol. Adding NAT, Pfsync, CARP and etc. can be useful in dynamic CC set-up. To improve PFF performance PF optimizer of OpenBSD can be employed, to optimize rule matching. 


\section{SELECTED STUdies}

Rexford, J. (2012). Programming languages for programmable networks. Proceedings of the 39th annual ACM SIGPLAN-SIGACT symposium on Principles of programming languages (POPL '12) (pp. 215-216). New York, NY, USA: ACM. doi:10.1145/2103656.2103683

Szefer, J., Keller, E., Lee, R. B., \& Rexford, J. (2011). Eliminating the hypervisor attack surface for a more secure Cloud. Proceedings of the 18th ACM conference on Computer and communications security - CCS '11, 401. doi:10.1145/2046707.2046754

Litty, L., \& Lie, D. (2011). Patch auditing in infrastructure as a service Clouds. ACM SIGPLAN Notices, 46(7), 145. doi:10.1145/2007477.1952702

Zhou, S. (VMware Inc.), (2010). Virtual Networking, 80-85

Basak, D., Toshniwal, R., Maskalik, S., \& Sequeira, A. (2010). Virtualizing networking and security in the Cloud. ACM SIGOPS Operating Systems Review, 44(4), 86. doi:10.1145/1899928.1899939

Riteau, P. (2011). Building Dynamic Computing Infrastructures over Distributed Clouds. 2011 IEEE International Symposium on Parallel and Distributed Processing Workshops and Phd Forum (pp. 2097-2100). IEEE. doi:10.1109/IPDPS.2011.386

Tsugawa, M., Riteau, P., Matsunaga, A., \& Fortes, J. (2010). User-level virtual networking mechanisms to support virtual machine migration over multiple Clouds. 2010 IEEE Globecom Workshops, 568-572. doi:10.1109/GLOCOMW.2010.5700384

Jarraya, Y., Eghtesadi, A., Debbabi, M., Zhang, Y., \& Makan, P. (2012). Formal verification of security preservation for migrating virtual machines in the Cloud. 14th international conference on Stabilization, Safety, and Security of Distributed Systems (SSS'12), Andréa W. Richa and Christian Schei, 111-125. doi:10.1007/978-3-642-33536-5_12

Jarraya, Y., Eghtesadi, A., Debbabi, M., Zhang, Y., \& Pourzandi, M. (2012). Cloud calculus: Security verification in elastic Cloud computing platform. 2012 International Conference on Collaboration Technologies and Systems (CTS), 447-454. doi:10.1109/CTS.2012.6261089

Tavakoli, Z., Meier, S., \& Vensmer, A. (2012). A Framework for Security Context Migration in a Firewall Secured Virtual Machine Environment. Information and Communication Technologies conference, 41-51.

Chen, X. W. and Y. (2011). Approach of the Secure Communication Mechanism for the OffSite Live Virtual Machine Migrations. In Advances in Computer Science, Intelligent System and Environment Advances in Intelligent and Soft Computing. Springer Berlin Heidelberg., 104, 767-773.

Cabuk, S. et al., 2008. The Rise and Rise of the Declarative Datacentre. Microsoft Research Cambridge, UK, May 12-13, pp.17-20.

Aslam, M., Gehrmann, C. \& Bjorkman, M., 2012. Security and Trust Preserving VM Migrations in Public Clouds. In 2012 IEEE 11th International Conference on Trust, Security and Privacy in Computing and Communications. Ieee, pp. 869-876.

Mann, V. et al., 2012. CrossRoads: Seamless VM mobility across data centers through software defined networking. In 2012 IEEE Network Operations and Management Symposium. Ieee, pp. 88-96.

Wu, H., \& Winer, C. (2010). Network Security for Virtual Machine in Cloud Computing. 5th International Conference on Computer Sciences and Convergence Information Technology (ICCIT), 2010 (pp. 18-21).

Miaol, Q. et al., 2010. Developing a Virtual Network Environment for Analyzing Malicious Network Behavior. In 2010 International Conference on Educational and Network Technology (ICENT 2010). Qinhuangdao, China: IEEE Comput. Soc, pp. 271-275. 
Dawoud, W., Takouna, I. \& Meinel, C., 2010. Infrastructure as a Service Security : Challenges and Solutions. In The 7th International Conference on Informatics and Systems. pp. 1-8.

Xianqin, C. et al., 2009. Seamless virtual machine live migration on network security enhanced hypervisor. 2009 2nd IEEE International Conference on Broadband Network \& Multimedia Technology, pp.847-853. 


\section{APPENDIX}

\section{Appendix A - KVM Live Migration}

1. Setup

- Start guest on destination, connect, enable dirty page logging and more

2. Transfer Memory

- Guest continues to run

- Bandwidth limitation (controlled by the user)

a First transfer the whole memory

- Iteratively transfer all dirty pages (pages that were written to by the guest).

3. Stop the guest

n And sync VM image(s) (guest's hard drives)

4. Transfer State

- As fast as possible (no bandwidth limitation)

n All VM devices' state and dirty pages yet to be transferred

5. Continue the guest

- On destination upon success

- Broadcast "I'm over here" Ethernet packet to announce new location of $\mathrm{NIC}(\mathrm{s})$.

n On source upon failure (with one exception)

Figure 10: KVM live migration algorithm 


\section{Appendix B - Research Methodology Alternative}

Table 12: Research methodology comparison

\section{Interview}

Less accessibility.

Respondents had to be selected from Karlskrona only for face-toface interviews. Going to other cities would be costly. 2 related respondents were found and interviewed.

Less related data available.

Selecting an appropriate interviewee is very important, as getting the relevant information is highly dependent on interviewee. Cloud VM security is not a specified designation in IT security or $\mathrm{CC}$, which is why the chances of selecting an inappropriate interviewee cannot be ignored. 1 respondent was found suitable.

Slow identification of related data/sources.

Appropriate respondent sample comprised of practitioners or professionals who can give firsthand information on research questions and who are well experienced in the domain i.e. concerned people from CPs.

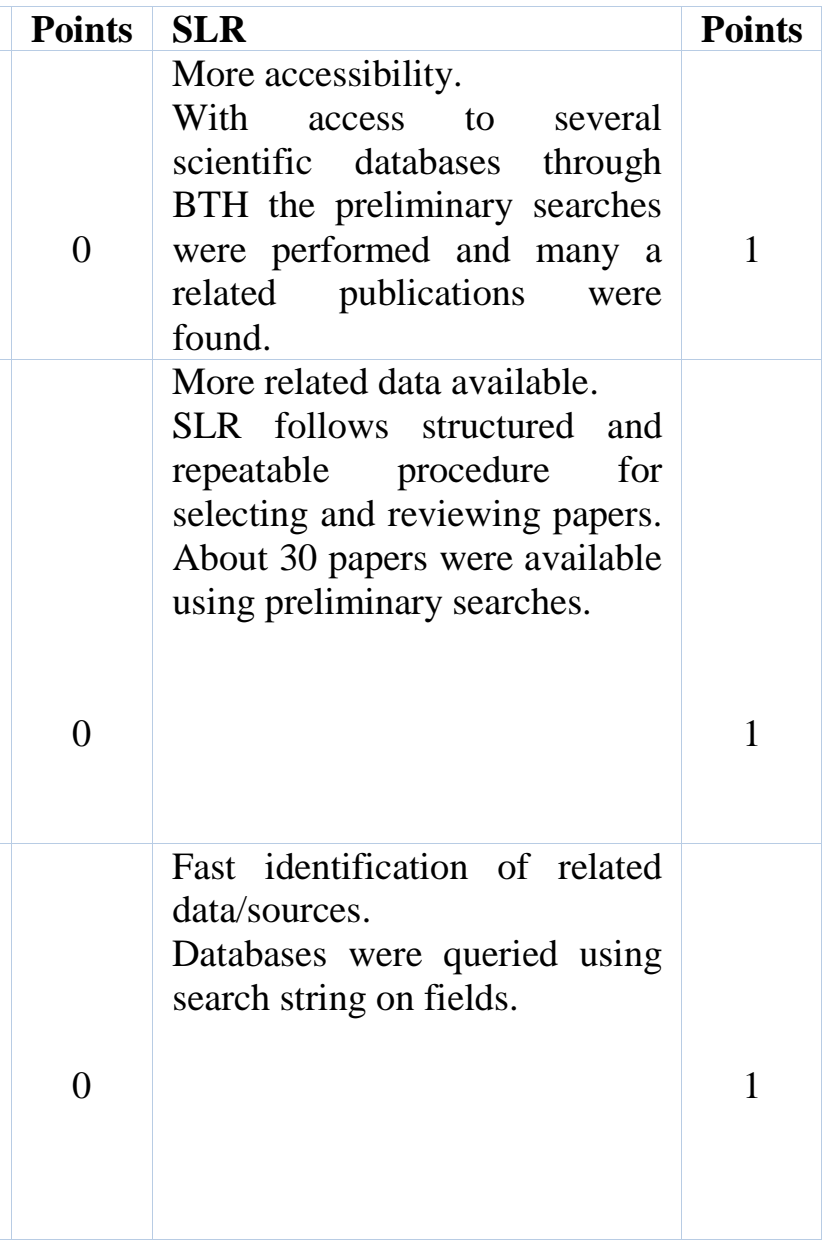




\section{Appendix C - SLR Search Strings}

ACM: (((Abstract:"virtual machine*") OR (Abstract:"Virtual Machine*") OR (Abstract:VM) OR (Abstract:"Virtual Network*") OR (Abstract:VN) (Abstract:"Hypervisor*") OR (Abstract:"hypervisor*") OR (Abstract:"Virtual Machine Monitor*") OR (Abstract:"virtual machine monitor*") OR (Abstract:"VMM*")) and ((Abstract:migrat*) OR (Abstract:transfer*) OR (Abstract:mobility)) and ((firewall*) OR (Firewall*)) and ((Cloud*) OR (Cloud*)))

CiteSeer: abstract:("virtual machine*" OR "VM*" OR "Virtual Machine*" OR "Virtual Network*" OR "virtual network*" OR "VN*" OR "VMM" OR "virtual machine monitor*" OR "hypervisor*") AND abstract:("migrat*" OR "mobility" OR "transfer*") AND text:("firewall*" OR "Firewall*") AND text:cloud*

Engineering Village: ((c((c)irtual machine*" OR "Virtual Machine*" OR VM* OR "Virtual Network*" OR VN* OR hypervisor* OR Hypervisor* OR "virtual machine monitor*" OR VMM OR "Virtual Machine Monitor*")) WN AB) AND (((firewall* OR Firewall*)) WN All fields)) AND (((Migrat* OR migrat* OR transfer* OR mobility)) WN AB)) AND (((Cloud* OR Cloud*)) WN All fields)), 1969-2012

IEEE: (("Abstract":"virtual machine*" OR "Abstract":VM OR "Abstract":"Virtual Network*" OR "Abstract":VN OR "Abstract":"Virtual Machine Monitor*" OR "Abstract":VMM OR "Abstract":"hypervisor" OR "Abstract":"hypervisors" OR "Abstract":"Hypervisor" OR "Abstract":"Hypervisors" OR "Abstract":"virtual machine monitor*") AND (Abstract:migration OR "Abstract":migrates OR "Abstract":migrate OR "Abstract":transfer* OR "Abstract":mobility) AND (firewalls OR firewall OR Firewalls OR Firewall) AND (Cloud OR Cloud OR Clouds or Clouds))

SCOPUS: (TITLE-ABS-KEY("Virtual Machine*") OR TITLE-ABS-KEY("virtual machine*") OR TITLE-ABS-KEY("VM*") OR TITLE-ABS-KEY("virtual network*") OR TITLE-ABS-KEY("VN*") OR TITLE-ABS-KEY("Hypervisor*") OR TITLE-ABSKEY("hypervisor*") OR TITLE-ABS-KEY("Virtual Machine Monitor*") OR TITLE-ABSKEY("virtual machine monitor*") OR TITLE-ABS-KEY("VMM*") OR TITLE-ABSKEY("Virtual Network*")) and (TITLE-ABS-KEY("migrat*") OR TITLE-ABSKEY("transfer*") OR TITLE-ABS-KEY("mobility")) AND (ALL("Firewall*") OR ALL("firewall*")) AND (ALL("Cloud*") OR ALL("Cloud*"))

ScienceDirect: (TITLE-ABSTR-KEY("Virtual Machine*") or TITLE-ABSTRKEY("virtual machine*") or TITLE-ABSTR-KEY("VM*") or TITLE-ABSTR-KEY("virtual network*") or TITLE-ABSTR-KEY("VN*") or TITLE-ABSTR-KEY("Hypervisor*") or TITLE-ABSTR-KEY("hypervisor*") or TITLE-ABSTR-KEY("Virtual Machine Monitor*") or TITLE-ABSTR-KEY("virtual machine monitor*") or TITLE-ABSTR-KEY("VMM*") or TITLE-ABSTR-KEY("Virtual Network*")) and (TITLE-ABSTR-KEY("migrat*") OR TITLEABSTR-KEY("transfer*") OR TITLE-ABSTR-KEY("mobility")) and ((Cloud) or (Cloud)) and (firewall* OR Firewall*)

Springerlink: '("virtual machine*" OR "Virtual Machine*" OR VM OR "Virtual Network*" OR "virtual network* OR VN" OR VMM or "virtual machine monitor*" OR "Virtual Machine Monitor*” OR "hypervisor*" OR "Hypervisor*”) and (migrat* OR transfer* OR mobility) and (Firewall* OR firewall*) and (Cloud* OR Cloud*))'

Wiley Inter Science: ("Virtual Machine*" OR "Virtual Network* OR "virtual network*" OR "virtual machine*" OR "Virtual Machine Monitor*" OR "virtual machine monitor*" OR "VMM" OR "Hypervisor*" OR "hypervisor*") in Abstract and (migrat* OR transfer* OR mobility) in Abstract and (firewall* OR Firewall*) in All Fields and (Cloud* OR Cloud*) in All Fields. 
Web of Knowledge: TS=(("virtual machine*" OR "Virtual Machine*" OR VM OR "Virtual Network*" OR "virtual network*" OR VN OR "hypervisor*" OR "virtual machine monitor*" OR "VMM")) and $\mathrm{TS}=(($ migrat* $))$ and $\mathrm{TS}=(($ Firewall* OR firewall* $))$ and $\mathrm{TS}=\left(\left(\right.\right.$ Cloud $^{*}$ OR Cloud $\left.\left.*\right)\right)$

\section{Appendix D - VF Implementation}

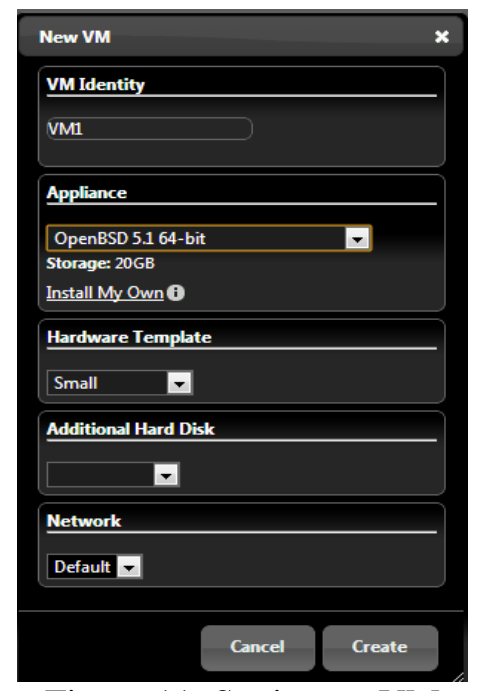

Figure 11: Setting up VM

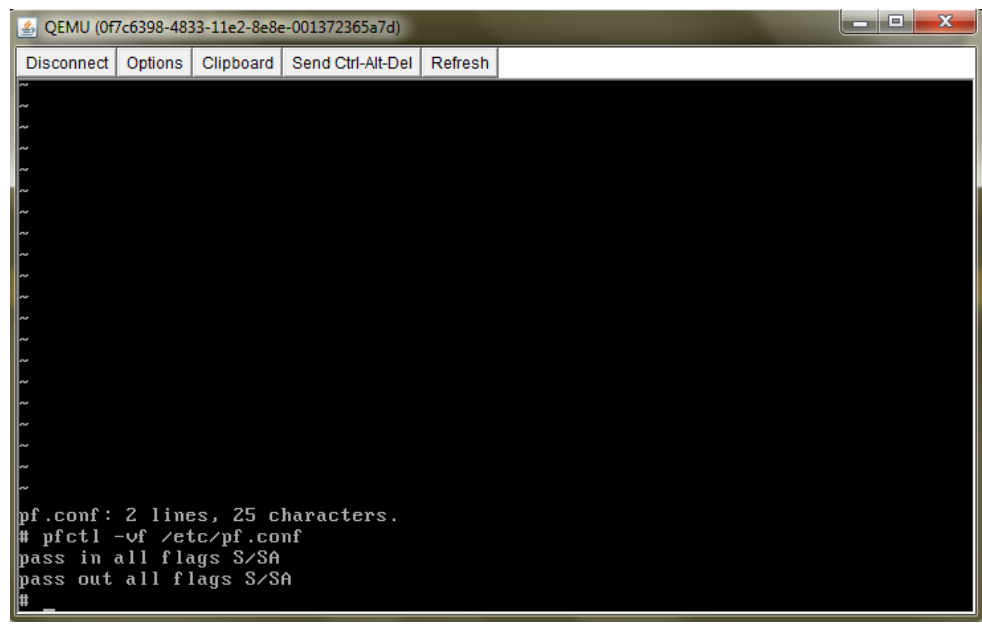

Figure 12: Remote VM firewall configuration

The default pass rules are set for VM. 


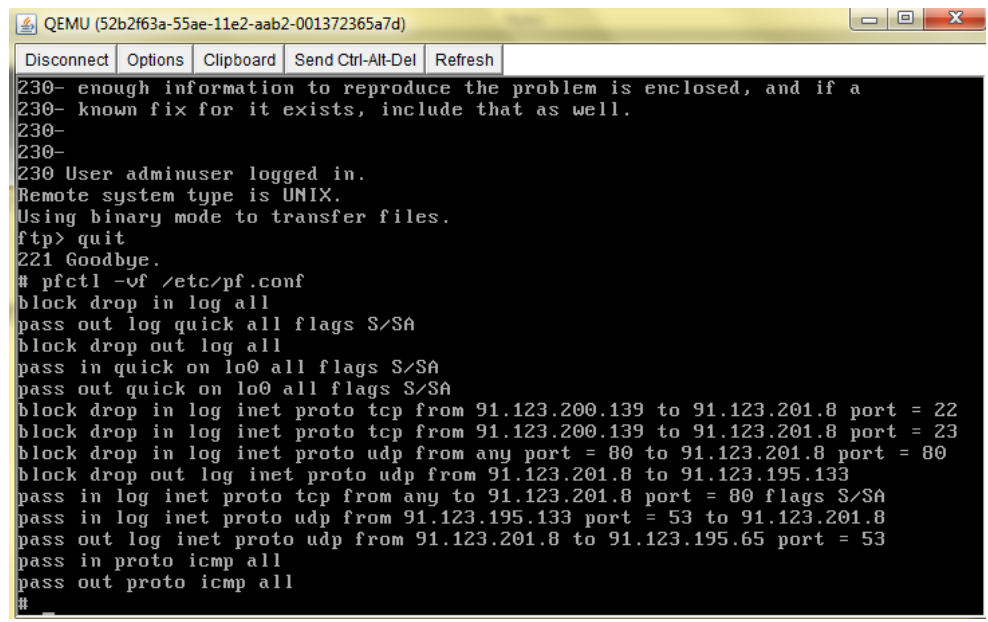

Figure 13: Target VM firewall configuration

It includes sample firewall rules.

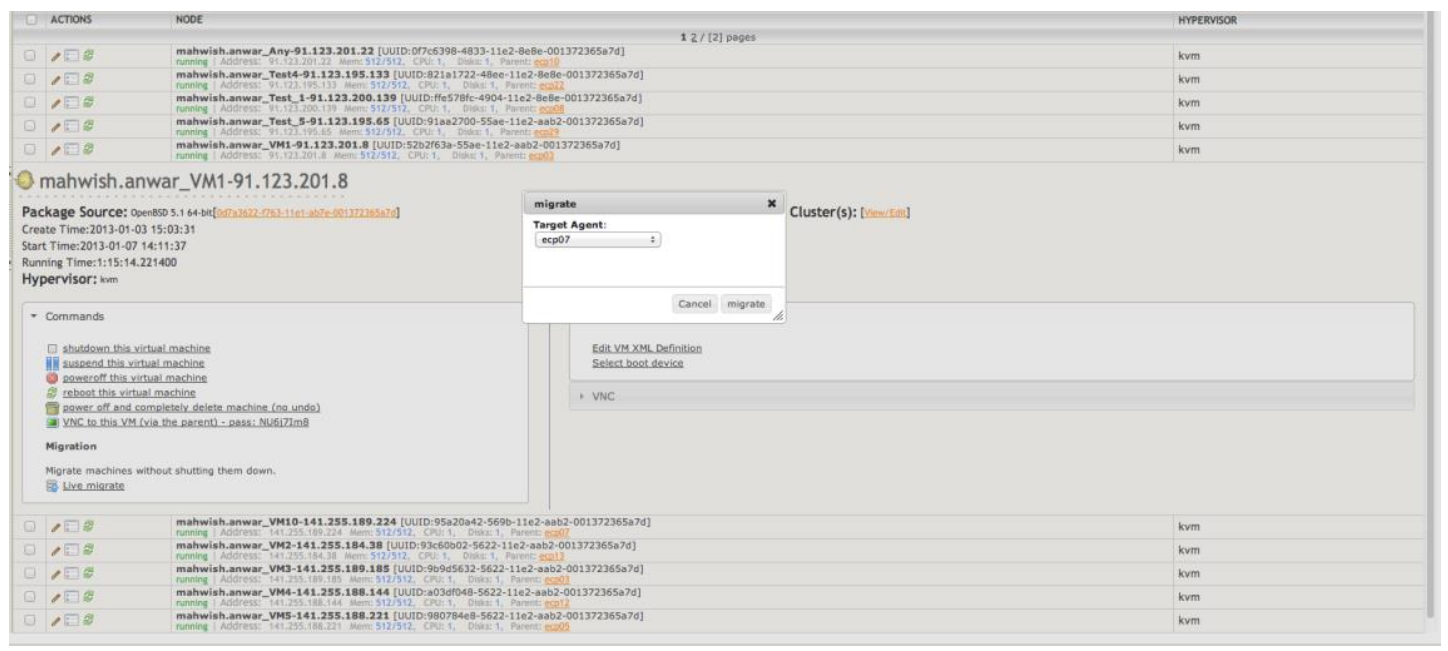

Figure 14: KVM Migration Interface

The figure depicts the VM name, its IP, along with other VM specifications. The migrations are executed using buttons. Other actions such as VM start, stop, delete or clone are all done using this administrative interface. 


\section{Appendix E - VF Rule Verification Before Migration}

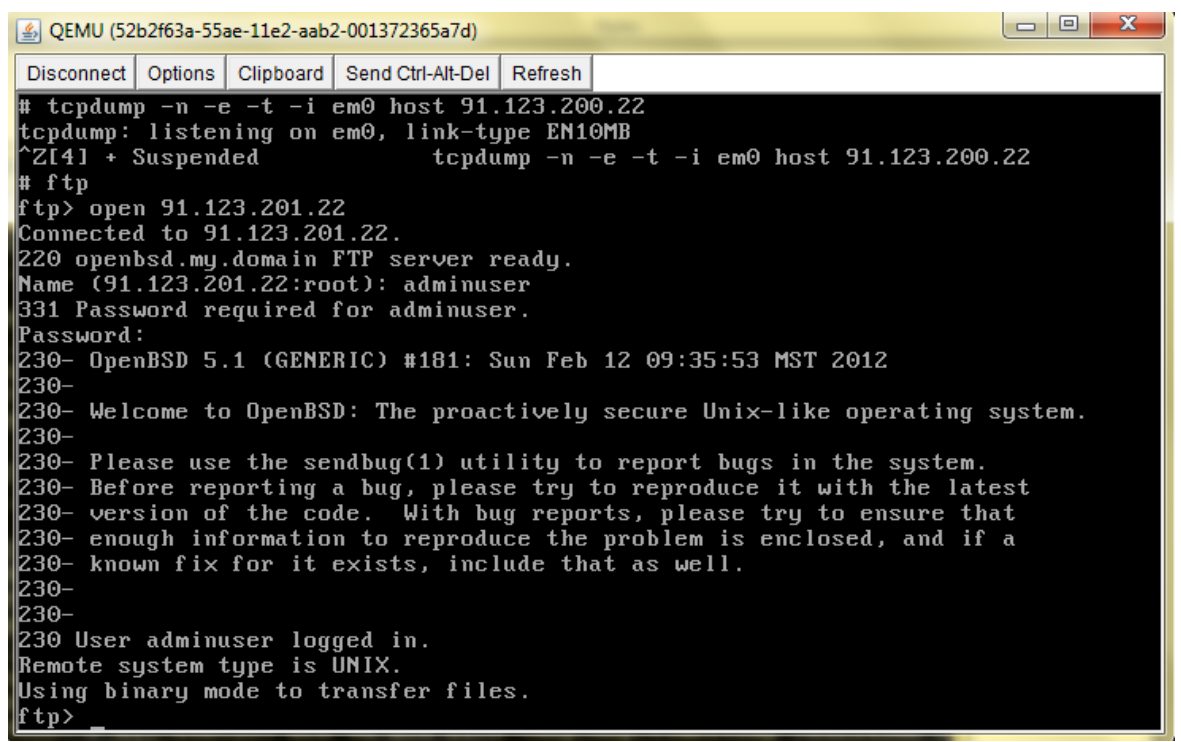

Figure 15: PFF rule verification before migration.

Verifying FTP rule between VM2 (IP: 91.123.201.8) and VM1 (IP: 91.123.201.22). FTP request was sent from VM2 to VM1. After user authentication the FTP establishes connection with VM1. The TCPDUMP $\log$ in bottom screen acknowledges the successful FTP connection.

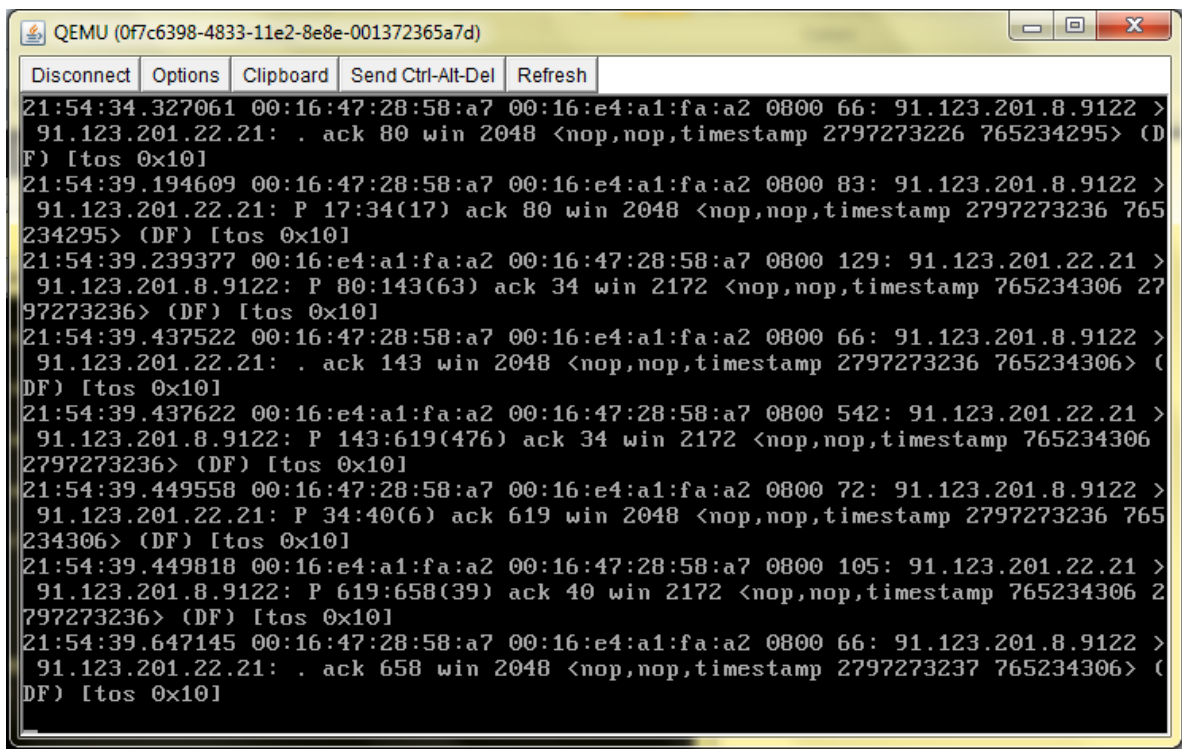

Figure 16: PFF rule verification before migration (log).

Verifying FTP rule between VM2 (IP: 91.123.201.8) and VM1 (IP: 91.123.201.22). FTP request was sent from VM2 to VM1. After user authentication the FTP establishes connection with VM1. The TCPDUMP log acknowledged the successful FTP connection. 


\section{Appendix F - VF Rule Verification After Migration}

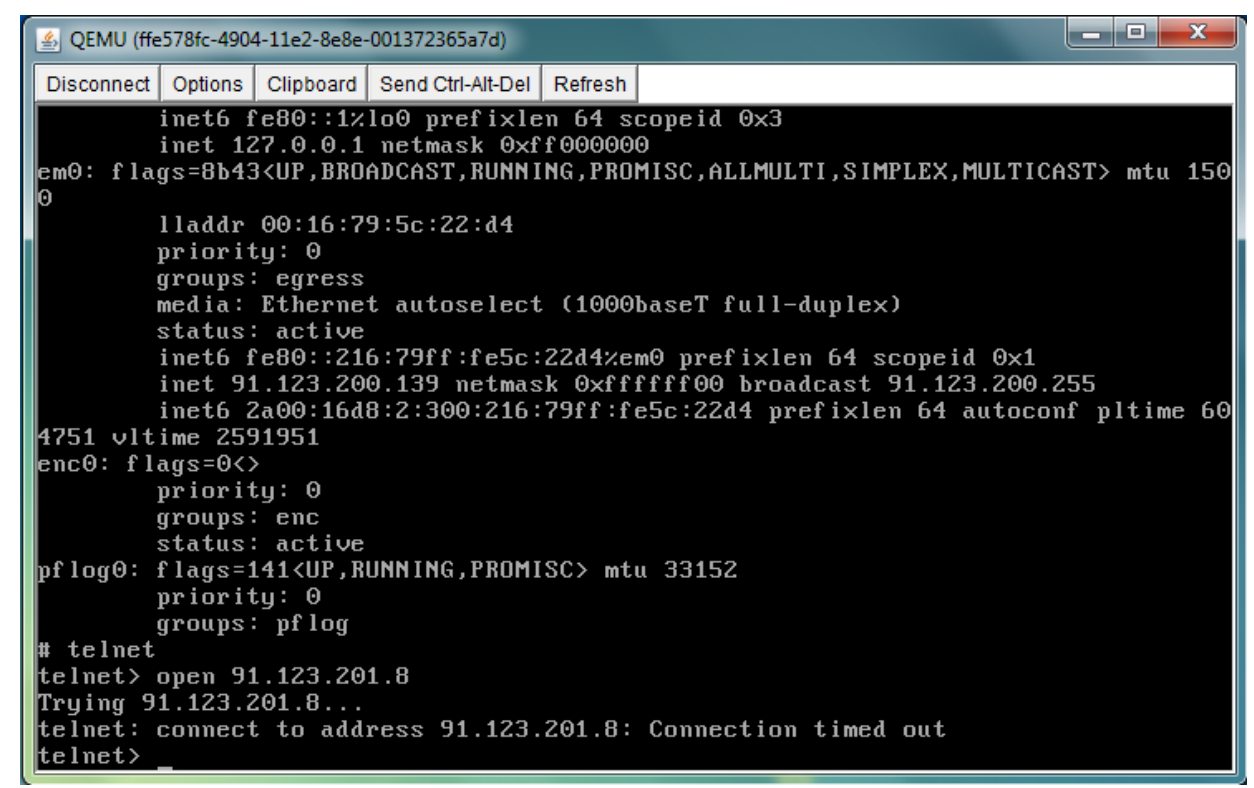

Figure 17: PFF rule verification after migration.

Verifying FTP rule between VM2 (IP: 91.123.201.8) and VM1 (IP: 91.123.201.22). FTP request was sent from VM2 to VM1. After user authentication FTP established connection with VM1. The TCPDUMP $\log$ in bottom screen acknowledges the successful FTP connection.

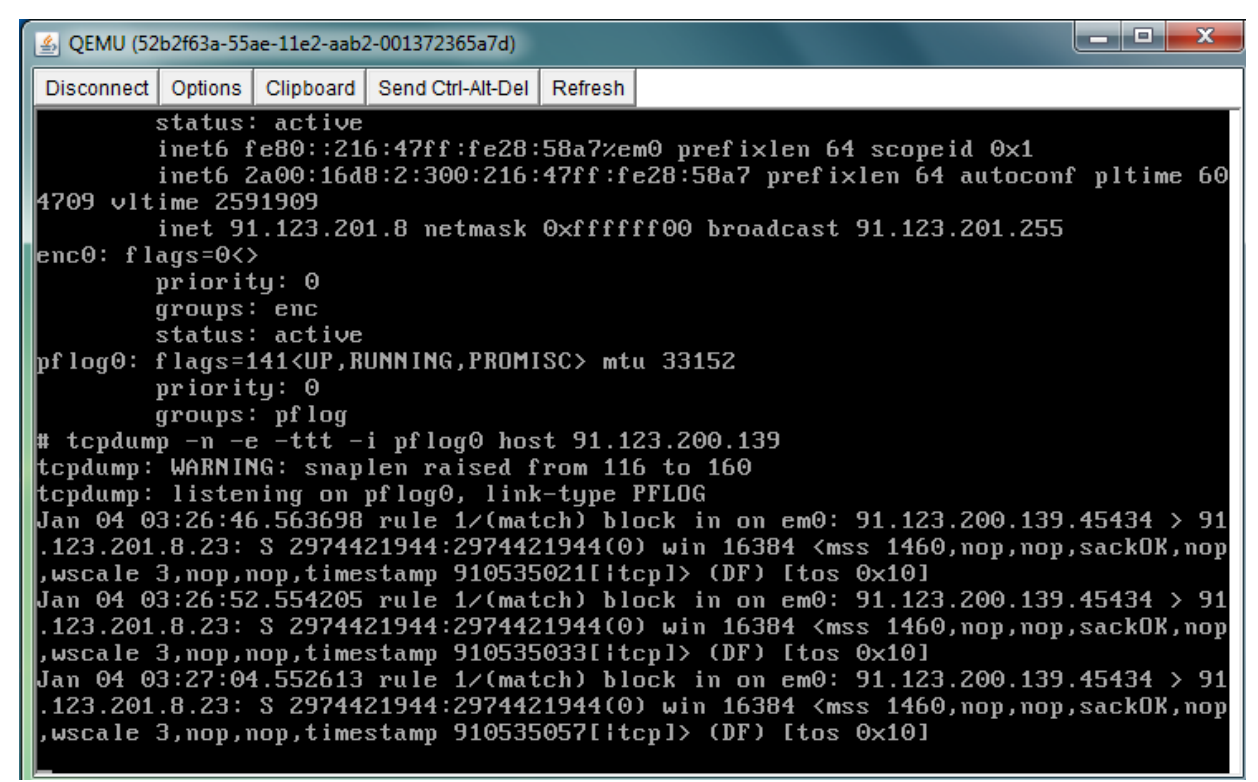

Figure 18: PFF rule verification after migration $(\log )$.

Verifying FTP rule between VM2 (IP: 91.123.201.8) and VM1 (IP: 91.123.201.22). FTP request was sent from VM2 to VM1. After user authentication the FTP establishes connection with VM1. TCPDUMP log acknowledged the successful FTP connection 


\section{REFERENCES}

Cisco, 2011. Cisco Cloud Security Accelerates Cloud Adoption, White Paper, Available at: http://www.cisco.com/en/US/solutions/collateral/ns170/ns896/ns1066/white_paper_c11 $-674558 . h t m l$.

Armbrust, M. et al., 2009. Above the Clouds : A Berkeley View of Cloud Computing. Science, 53(UCB/EECS-2009-28), pp.07-013.

Artymiak, J., 2003. Building Firewalls with OpenBSD and PF 2nd ed., Lublin.

Aslam, M., Gehrmann, C. \& Bjorkman, M., 2012. Security and Trust Preserving VM Migrations in Public Clouds. In 2012 IEEE 11th International Conference on Trust, Security and Privacy in Computing and Communications. Ieee, pp. 869-876.

Barbara, K. (Keele U. and U. of D., 2007. Guidelines for performing Systematic Literature Reviews in Software Engineering.

Basak, D. et al., 2010. Virtualizing networking and security in the cloud. ACM SIGOPS Operating Systems Review, 44(4), p.86.

Bellovin, S.M., 2006. Virtual Machines, Virtual Security?, Communications of the ACM, 49(10), p.1987.

Cabido, P., 2011. Packet Filtering - The first line of defense. , pp.1-10.

Cabuk, S. et al., 2008. The Rise and Rise of the Declarative Datacentre. Microsoft Research Cambridge, UK, May 12-13, pp.17-20.

Carlin, S. \& Curran, K., 2011. Cloud Computing Security. International Journal of Ambient Computing and Intelligence, 3(1), pp.14-19.

Catteddu, D. \& Hogben, G., 2009. The European Network and Information Security Agency (ENISA) This work takes place in the context of ENISA's Emerging and Future Risk programme.

Chen, X.W. and Y., 2011. Approach of the Secure Communication Mechanism for the OffSite Live Virtual Machine Migrations. In Advances in Computer Science, Intelligent System and Environment Advances in Intelligent and Soft Computing. Springer Berlin Heidelberg., 104, pp.767-773.

Christine B. Feak, J.M.S., 2009. Telling a research story: writing a literature review. revised/ex.

Clark, C. et al., 2005. Live migration of virtual machines. Proceedings of the 2nd Symposium on Networked Systems Design \& Implementation (NSDI), 2(Vmm), pp.273-286.

Creswell, J.W., 2009. The Selection of a Research Design. In Research Design Qualitative, Quantitative, and Mixed Methods Approaches. California: SAGE, pp. 16-18.

Cutsem, T. Van et al., 2004. On the Performance of SOAP in a Non-Trivial Peer-to-Peer Experiment. In Component Deployment: Second International Working Conference (LNCS volume 3083/2004). p. 14.

Dawoud, W., Takouna, I. \& Meinel, C., 2010. Infrastructure as a Service Security: Challenges and Solutions. In The 7th International Conference on Informatics and Systems. pp. 1-8.

Decusatis, C.J.S. et al., 2012. Communication within Clouds : Open Standards and Proprietary Protocols for Data Center Networking. , (September), pp.26-33.

Garber, L., 2012. The Challenges of Securing the Environment. , (January), pp.17-20.

Garrison, B.G., Kim, S. \& Wakefield, R.L., 2012. Success Factors for Deploying Cloud. Communications of the ACM, 55(9), pp.62-68.

Golnabi, K. et al., 2006. Analysis of Firewall Policy Rules Using Data Mining Techniques. 2006 IEEE/IFIP Network Operations and Management Symposium NOMS 2006, pp.305-315.

Greene, T., 2009. Cloud Security Stokes Concerns at RSA. Available at: http://www.networkworld.com/news/2009/042309-rsa-cloud-security.html. 
Haletky, E.L., 2008. Are Virtual Firewalls a Real Solution for VM Security? Available at: http://www.cio.com/article/439018/Are_Virtual_Firewalls_a_Real_Solution_for_VM_ Security_.

Hao, F. et al., 2009. Enhancing dynamic cloud-based services using network virtualization. Proceedings of the 1st ACM workshop on Virtualized infrastructure systems and architectures - VISA '09, p.37.

Ingham, K. \& Forrest, S., 2002. A History and Survey of Network Firewalls.

Jarraya, Y., Eghtesadi, A., et al., 2012. Cloud calculus: Security verification in elastic cloud computing platform. 2012 International Conference on Collaboration Technologies and Systems (CTS), pp.447-454.

Jarraya, Y., Eghtesadi, Arash, et al., 2012. Formal verification of security preservation for migrating virtual machines in the cloud. 14th international conference on Stabilization, Safety, and Security of Distributed Systems (SSS'12), Andréa W. Richa and Christian Schei, pp.111-125.

Kofod-petersen, A., 2012. How to do a Structured Literature Review in computer science., pp.1-9.

Kourai, K., Azumi, T. \& Chiba, S., 2012. A Self-Protection Mechanism against SteppingStone Attacks for IaaS Clouds. 2012 9th International Conference on Ubiquitous Intelligence and Computing and 9th International Conference on Autonomic and Trusted Computing, pp.539-546.

Li, H.-C. et al., 2010. Analysis on Cloud-Based Security Vulnerability Assessment. 2010 IEEE 7th International Conference on E-Business Engineering, pp.490-494.

Litty, L. \& Lie, D., 2011. Patch auditing in infrastructure as a service clouds. ACM SIGPLAN Notices, 46(7), p.145.

Mann, V. et al., 2012. CrossRoads: Seamless VM mobility across data centers through software defined networking. In 2012 IEEE Network Operations and Management Symposium. Ieee, pp. 88-96.

Mell, P. \& Grance, T., 2011. The NIST Definition of Cloud Computing: Recommendations of the National Institute of Standarts and Technology. National Institute of Standards and Technology.

Miaol, Q. et al., 2010. Developing a Virtual Network Environment for Analyzing Malicious Network Behavior. In 2010 International Conference on Educational and Network Technology (ICENT 2010). Qinhuangdao, China: IEEE Comput. Soc, pp. 271-275.

Miyamoto, T., Hayashi, M. \& Tanaka, H., 2009. Customizing Network Functions for High Performance Cloud Computing. In 2009 Eighth IEEE International Symposium on Network Computing and Applications. Ieee, pp. 130-133.

Modi, C. et al., 2012. A survey on security issues and solutions at different layers of Cloud computing. The Journal of Supercomputing.

Northcutt, S. (The Sans Technology Instutute), 2009. Interview: Amir Ben-Efraim, CEO, Altor Networks. Available at: http://www.sans.edu/research/securitylaboratory/article/benefraim-thought [Accessed January 5, 2013].

Oberheide, J., Cooke, E. \& Jahanian, F., 2008. Empirical Exploitation of Live Virtual Machine Migration, Michigan.

Patton, S., Doss, D. \& Yurcik, W., 2000. Open source versus commercial firewalls: functional comparison. Proceedings 25th Annual IEEE Conference on Local Computer Networks. LCN 2000, pp.223-224.

Qina, Z., Zhangc, Q. \& Wana, C., 2012. State-of-the-art Virtualization Security in Cloud Computing. Journal of Information \& Computational Science, 6(June), pp.1487-1497.

Ranjith, P., Priya, C. \& Shalini, K., 2012. On covert channels between virtual machines. Journal in Computer Virology, 8(3), pp.85-97.

Rexford, J., 2012. Programming languages for programmable networks. In Proceedings of the 39th annual ACM SIGPLAN-SIGACT symposium on Principles of programming languages (POPL '12). New York, NY, USA: ACM, pp. 215-216. 
Riteau, P., 2011. Building Dynamic Computing Infrastructures over Distributed Clouds. In 2011 IEEE International Symposium on Parallel and Distributed Processing Workshops and Phd Forum. IEEE, pp. 2097-2100.

Ryan, M. (The Sans Institute), 2002. OpenBSDs PF: An alternative to Commercial Firewalls. As part of GIAC practical repository.

Salfner, F., Tr, P. \& Polze, A., 2011. Downtime Analysis of Virtual Machine Live Migration. The Fourth International Conference on Dependability (DEPEND 2011), pp.100-105.

Scarfone, K. \& Hoffman, P., 2009. Guidelines on Firewalls and Firewall Policy: Recommendations of the National Institute of Standards and Technology,

Schunter, M. \& Eriksson, K., 2011. Automated Information Flow Analysis of Virtualized Infrastructures. 16th European Symposium on Research in Computer Security, ESORICS 2011, 6879 LNCS, pp.392-415.

Shetty, J., M R, A. \& G, S., 2012. A Survey on Techniques of Secure Live Migration of Virtual Machine. International Journal of Computer Applications, 39(12), pp.34-39. Available at: http://research.ijcaonline.org/volume39/number12/pxc3877305.pdf.

Sqalli, M.H., Al-Haidari, F. \& Salah, K., 2011. EDoS-Shield - A Two-Steps Mitigation Technique against EDoS Attacks in Cloud Computing. 2011 Fourth IEEE International Conference on Utility and Cloud Computing, pp.49-56.

Stabler, G. et al., Elastic IP and Security Groups Implementation Using OpenFlow Categories and Subject Descriptors. , pp.53-60.

Szefer, J. et al., 2011. Eliminating the hypervisor attack surface for a more secure cloud. Proceedings of the 18th ACM conference on Computer and communications security CCS '11, p.401.

Tavakoli, Z., Meier, S. \& Vensmer, A., 2012. A Framework for Security Context Migration in a Firewall Secured Virtual Machine Environment. Information and Communication Technologies Conference, pp.41-51.

Timothy Broomhead, Laurence Cremean, Julien Ridoux, and D.V., 2010. Virtualize everything but time. In 9th USENIX conference on Operating systems design and implementation (OSDI'10). CA, USA: USENIX Association, Berkeley, pp. 1-6.

Tripathi, S. et al., 2009. Crossbow Virtual Wire : Network in a Box.

Tsugawa, M. et al., 2010. User-level virtual networking mechanisms to support virtual machine migration over multiple clouds. 2010 IEEE Globecom Workshops, pp.568572.

Wohlin, Per Runeson, Martin Höst, Magnus C. Ohlsson, Bjöorn Regnell, Anders Wesslén, C., 2000. Planning. In Experimentation in Software Engineering: An Introduction. Norwell, MA, USA: Kluwer Academic Publishers, pp. 47-49.

Wood, T. et al., 2007. Black-box and Gray-box Strategies for Virtual Machine Migration. In 4th USENIX Symposium on Networked Syatems Design \& Implementation (NSDI). USENIX Association, pp. 229-242.

Wood, T. \& Shenoy, P., 2010. CloudNet : A Platform for Optimized WAN Migration of Virtual Machines, Massachusetts.

Wood, T. \& Shenoy, P., 2011. CloudNet: Dynamic Pooling of Cloud Resources by Live WAN Migration of Virtual Machines. , pp.121-132.

Wu, H. \& Winer, C., 2010. Network Security for Virtual Machine in Cloud Computing. In 5th International Conference on Computer Sciences and Convergence Information Technology (ICCIT), 2010. pp. 18-21.

Xianqin, C. et al., 2009. Seamless virtual machine live migration on network security enhanced hypervisor. 2009 2nd IEEE International Conference on Broadband Network \& Multimedia Technology, pp.847-853.

Xu, Z. et al., 2011. WAVNet: Wide-Area Network Virtualization Technique for Virtual Private Cloud. 2011 International Conference on Parallel Processing, pp.285-294.

Zaborovsky, V. et al., 2011. Dynamic access control in cloud services. 2011 IEEE International Conference on Systems, Man, and Cybernetics, pp.1400-1404. 
Zhang, H., Babar, M.A. \& Tell, P., 2011. Identifying relevant studies in software engineering. Information and Software Technology, 53(6), pp.625-637.

Zhou, S. (VMware Inc), 2010. Virtual Networking. , pp.80-85. 